Revue des patrimoines

29 | 2016

Ensembles mobiliers, industriels, techniques.

Connaissance, protection, conservation, présentation au public

\title{
Regard sur les ensembles mobiliers civils bourguignons
}

Some collections of civil movable objects in the Burgundy region

Anne-Bénédicte Clert, Bruno François, Adeline Rivière et Michaël Vottero

\section{(2) OpenEdition}

Journals

Édition électronique

URL : http://journals.openedition.org/insitu/13548

DOI : 10.4000/insitu. 13548

ISSN : $1630-7305$

Éditeur

Ministère de la culture

Référence électronique

Anne-Bénédicte Clert, Bruno François, Adeline Rivière et Michaël Vottero, « Regard sur les ensembles mobiliers civils bourguignons », In Situ [En ligne], 29 | 2016, mis en ligne le 14 octobre 2016, consulté le 19 avril 2019. URL : http://journals.openedition.org/insitu/13548 ; DOI : 10.4000/insitu.13548

Ce document a été généré automatiquement le 19 avril 2019

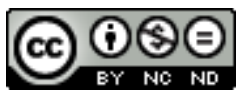

In Situ Revues des patrimoines est mis à disposition selon les termes de la licence Creative Commons Attribution - Pas d'Utilisation Commerciale - Pas de Modification 4.0 International. 


\title{
Regard sur les ensembles mobiliers civils bourguignons
}

\author{
Some collections of civil movable objects in the Burgundy region
}

Anne-Bénédicte Clert, Bruno François, Adeline Rivière et Michaël Vottero

1 Riche de ses églises romanes, de ses châteaux et de ses hôpitaux et hôtels-Dieu, la Bourgogne conserve également de nombreux objets mobiliers protégés au titre des monuments historiques. Si 2237 immeubles sont aujourd'hui classés ou inscrits, près de 15000 objets le sont également. À l'image du territoire national, la protection en matière d'objets mobiliers concerne un vaste ensemble d'œuvres d'art religieuses qui relèvent des plus anciennes mesures de protection. Il convient également de noter que si certains objets, protégés au titre des monuments historiques, se trouvent en dehors d'églises, ce sont, pour un grand nombre d'entre eux, des œuvres religieuses liées à des décors d'édifices anciens. De nombreuses administrations se sont en effet installées dans des couvents et abbayes au lendemain de la Révolution.

2 D'abord consacrée à des œuvres d'art emblématiques de l'histoire de France ou d'artistes réputés, la protection au titre des monuments historiques s'est progressivement intéressée à des objets civils, dans certains châteaux notamment ${ }^{1}$. Suivant l'évolution de la doctrine en matière de protection et l'intérêt pour de nouveaux domaines, on assiste à partir des années 1970-1980 à une multiplication de protections qui dépassent la sphère religieuse : mobilier civil, objets médicaux, machines industrielles... un travail d'étude et de protection du patrimoine civil qui se poursuit en Bourgogne au moment où le patrimoine $\mathrm{du} \mathrm{xx}^{\mathrm{e}}$ siècle fait l'objet d'une vaste campagne de protection au titre des monuments historiques.

3 Les récolements des œuvres classées et inscrites, la plupart du temps conservées dans les églises, conduisent les conservateurs des antiquités et objets d'art à inspecter, lors de leurs missions de terrain, les mairies et édifices publics. Des urnes républicaines, des bannières laïques, des horloges, des luminaires... sont ainsi repérés et font l'objet, lorsqu'ils présentent un intérêt d'art et d'histoire suffisant, d'une proposition de 
protection. Des ensembles apparaissent également, tels les mobiliers des salles de conseil, comme celui de l'hôtel de ville de Sens (inscrit au titre des monuments historiques pour ses façades et toitures par arrêté du 30 août $1995^{2}$ ), qui devrait faire l'objet d'une campagne de protection, ou les riches collections de l'hôtel de ville de Vézelay présentées ci-dessous. Des thématiques apparaissent également. Ainsi, tandis que la conservation des antiquités et objets d'art de l'Yonne s'est intéressée aux préfectures et sous-préfectures, au patrimoine de la Grande Guerre, celle de Côte-d'Or s'est tournée vers les mobiliers de théâtre, celui de Dijon notamment.

4 Parmi les ensembles mobiliers les plus importants de la région figurent également ceux des hôpitaux ${ }^{3}$. Concernés de longue date par des protections, à l'image de l'hôtel-Dieu de Beaune, les centres hospitaliers de Bourgogne conservent de très riches collections. Audelà des objets d'art, la protection s'intéresse aujourd'hui aux objets et mobiliers de soin, en particulier ceux des $\mathrm{XIX}^{\mathrm{e}}$ et $\mathrm{Xx}^{\mathrm{e}}$ siècles. Toutefois, l'une des questions usuelles est celle du statut, public ou privé, de ces collections, à laquelle la conservation des antiquités et objets d'art de Côte-d'Or tente de répondre dans cet article.

Enfin, les réformes récentes de cartes administratives, les concentrations de services ont conduit depuis plusieurs années au déménagement d'administrations civiles: palais de justice, rectorats, gendarmeries... entraînant la question de la conservation des collections qu'elles peuvent contenir, la difficulté demeurant celle de la gestion de ces ensembles mobiliers après le départ de ces organismes de leurs sites historiques. Des protections, réalisées en amont, tentent d'y remédier, à l'image des ensembles mobiliers du palais de justice de Dijon. Ce sont ainsi de nouveaux terrains d'étude qui apparaissent régulièrement et qui viennent illustrer la variété du patrimoine bourguignon.

\section{Les collections municipales, des ensembles fragiles}

6 Les mairies, en dehors de certains hôtels de ville fastueux, ne sont pas, dans l'imaginaire collectif, des lieux abritant des collections remarquables. La modernisation des administrations provoque toutefois la disparition de nombreux meubles et objets courants. De même, dans les petites villes de campagne, la mairie fait souvent office de musée ou de dépôt d'objets liés à l'histoire de la commune. Des œuvres isolées apparaissent, telle la bannière de la ferme-école de L'Orme du Pont, conservée dans la mairie de Sainte-Colombe-sur-Loing (Yonne), prochainement classée (fig. 1), ou l'oriflamme du ballon monté Le Davy, à la mairie de Fussey (Côte-d'Or), classé par arrêté du 7 avril 2014 (fig. 2). 
Figure 1

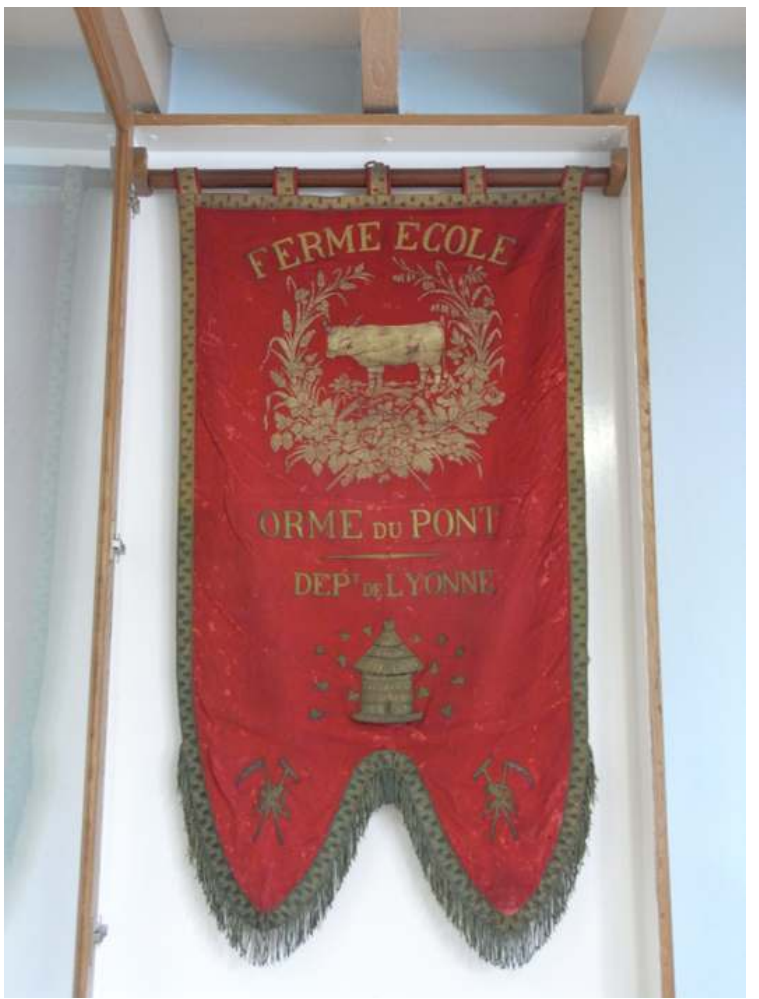

Anonyme, Bannière de la ferme école de l'Orme-du-Pont, Sainte-Colombe-sur-Loing, Mairie. (C) CAOA de I'Yonne. 
Figure 2

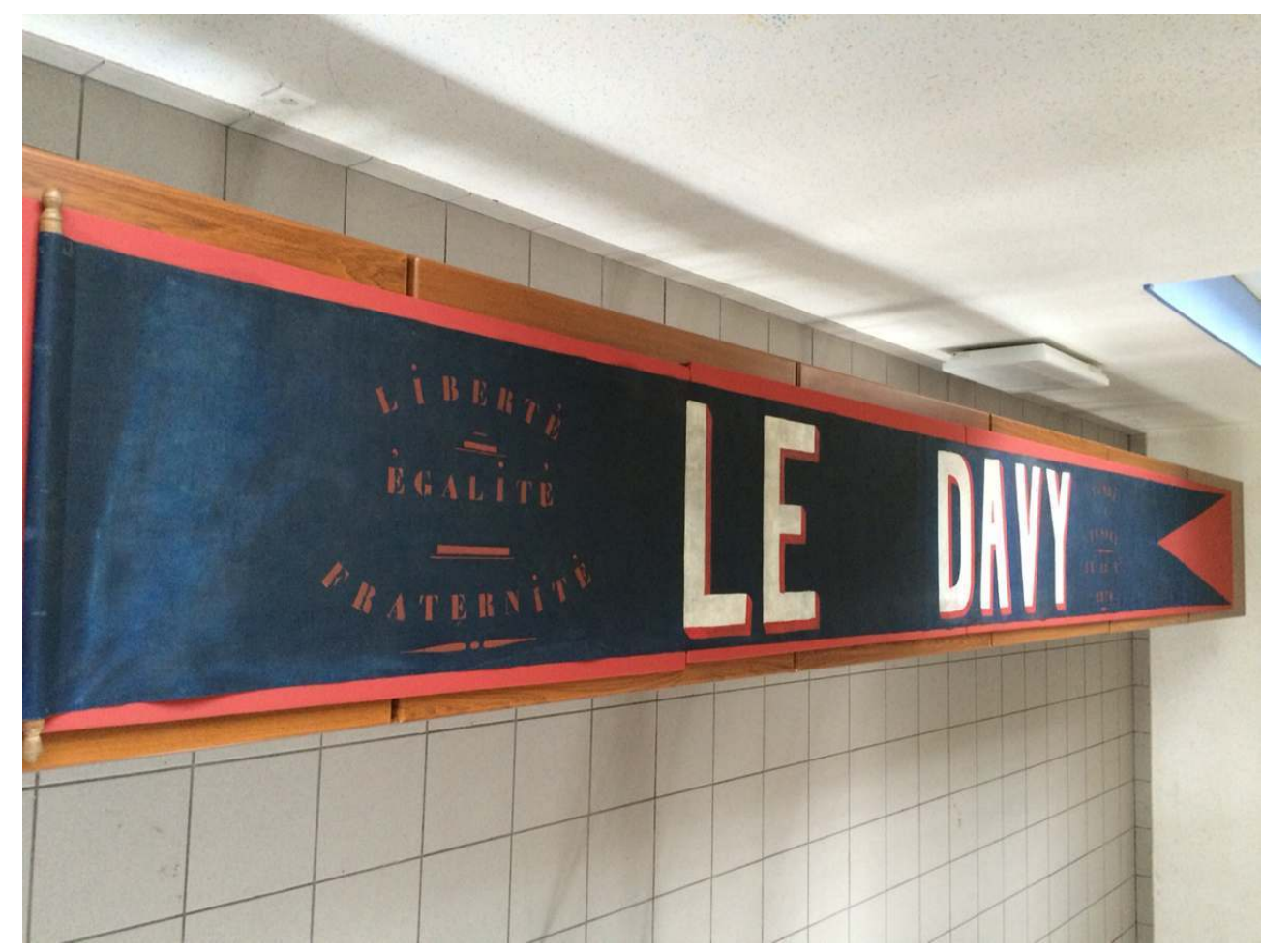

Anonyme, Oriflamme du ballon monté le Davy, 1870, Fussey, Mairie.

(c) CAOA de Côte-d'Or.

7 La récente enquête conduite par la conservation régionale des Monuments historiques et certaines conservations des antiquités et objets d'art sur la Première Guerre mondiale a permis de repérer quelques vestiges de cette période (fusils d'école, panneaux de camps américains...) (fig. 3, fig. 4). Les ensembles mobiliers de mairies demeurent encore rares. Si les cheminées et les pendules de l'hôtel de ville de Dijon sont classées depuis longtemps et le mobilier créé pour celui de Sens au XIX siècle prochainement proposé à la protection, l'une des rares mairies bourguignonnes à conserver un ensemble mobilier important est celle de Vézelay. En effet, connue pour sa célèbre basilique, la commune possède une mairie remarquable installée dans un hôtel particulier dont les collections, léguées à la Ville, ont été pour une grande partie classées, après les travaux d'AnneBénédicte Clert, conservateur délégué des antiquités et objets d'art de l'Yonne. 
Figure 3

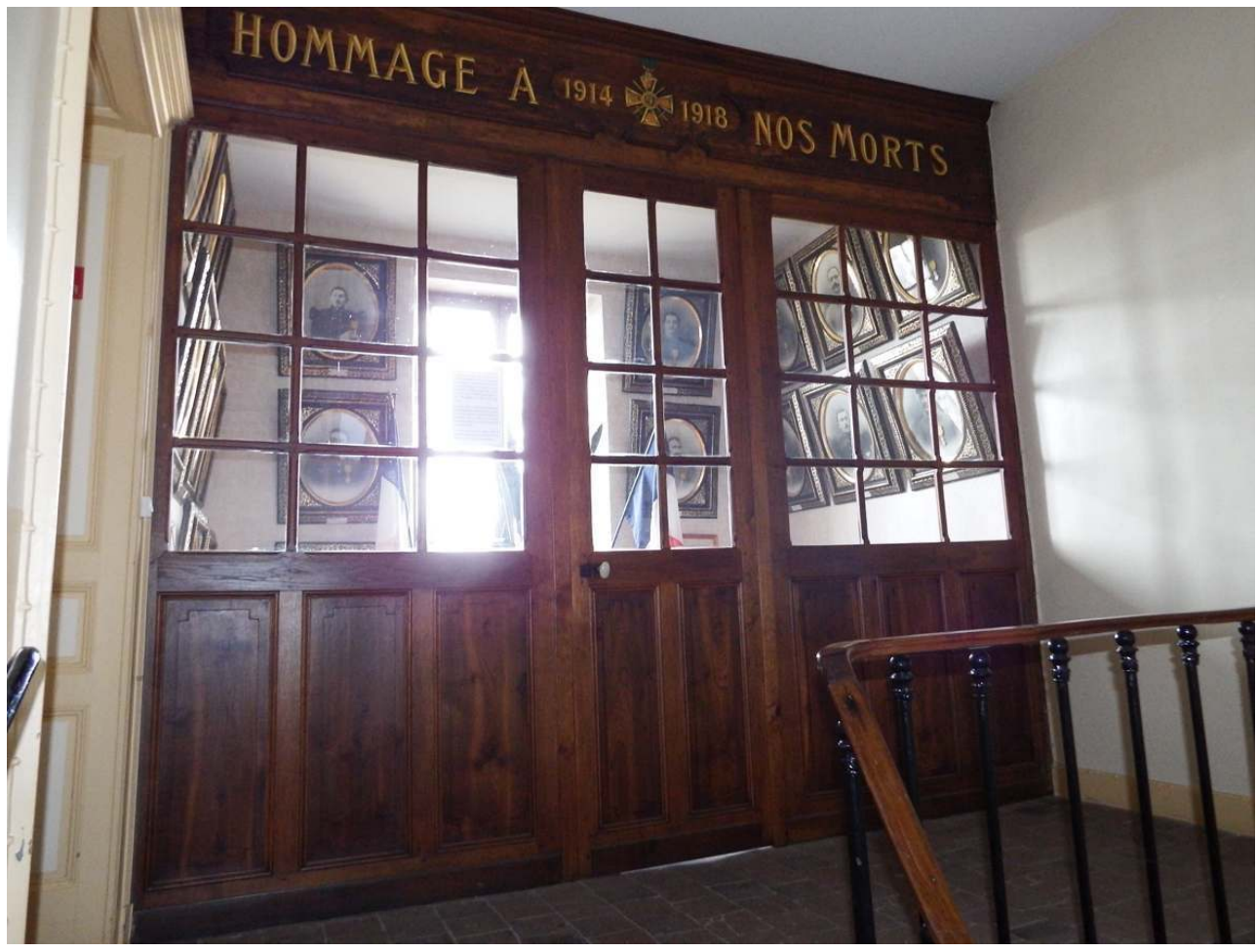

Anonyme, Salle d'honneur, 1920, Arces-Dilo, Mairie.

(c) CAOA de I'Yonne. 
Figure 4

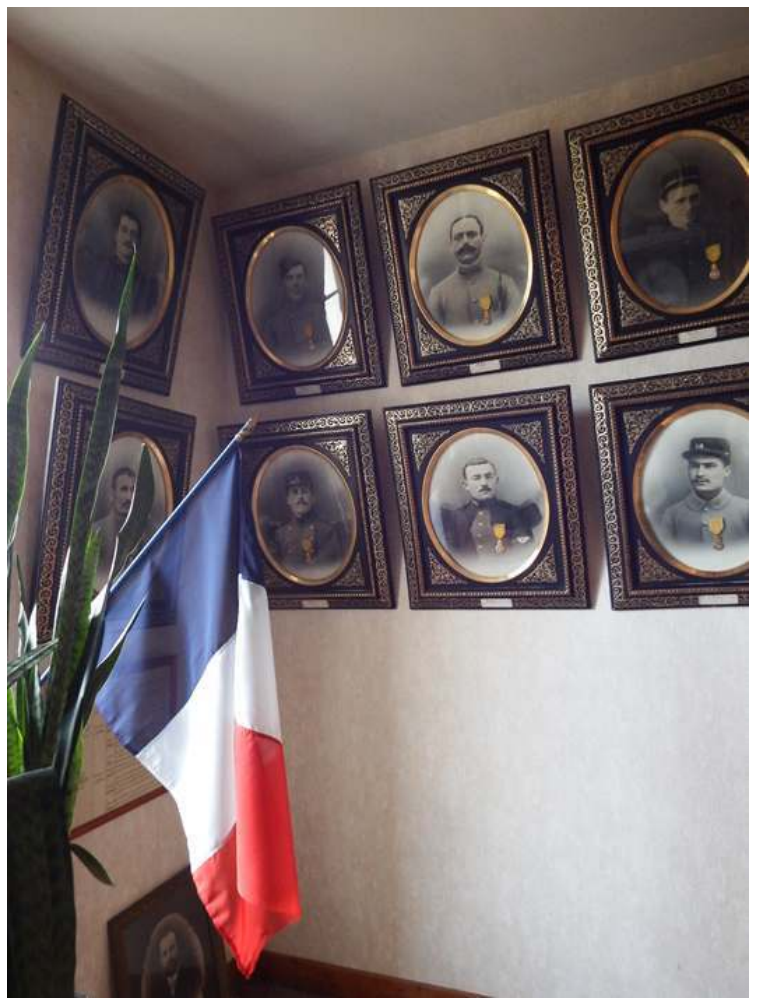

Anonyme, Salle d'honneur, 1920, Arces-Dilo, Mairie.

(c) CAOA de I'Yonne.

L'actuelle mairie de Vézelay est située rue Saint-Pierre, en face de l'ancienne église du même nom détruite en 1804 et dont l'emplacement fut converti en marché. C'est aujourd'hui la place Borot. Le pavillon à droite de la grande cour d'entrée était un rendezvous de chasse construit par François Friant, originaire du Nord, secrétaire particulier ou intendant du maréchal Vauban (1633-1707) qui y séjourna lui-même à plusieurs reprises ${ }^{5}$. Grâce au versement aux archives départementales de l'Yonne et à leur classement ainsi qu'au développement des instruments de recherche, de nouvelles sources ont pu être exploitées pour compléter l'histoire de ce bâtiment et en connaître les différents propriétaires.

En 1796, Gaspard Étienne Flandin (1767-1804) se dit «acquéreur de la maison appelée vulgairement Le Bicêtre ", autre nom donné à l'actuelle maison, siège de l'administration communale ${ }^{6}$. En 1813, Mme veuve Gaspard Flandin quitte cette maison, vendue par ses héritiers le 13 avril $1844^{7}$ à Hubert Borot (1800-1868), notaire à Villeneuve-Saint-Georges (Val-de-Marne) qui s'était retiré à Vézelay, dont il fut maire de 1854 à 1868. Vers 1853, il réalise " une construction nouvelle » sur la parcelle de la maison ${ }^{8}$. Victor Adrien Charles Hubert Borot, fils d'Hubert, et son épouse, ont étendu lentement les limites de leur propriété en achetant cinq ou six maisons qu'ils ont fait démolir et sur lesquelles ont été créés les jardins. À son décès, le 8 janvier 1925, Mme veuve Adrien Borot, née Marie Sophie Élisabeth Delaporte, lègue sa maison ainsi que les annexes, y compris celles acquises récemment, à la commune de Vézelay, sous réserve d'y installer « l'hôtel de ville, le musée et la bibliothèque ». Cette donation s'accompagne d'une somme de 300000 francs pour entretenir la propriété9. 
10 L'intérieur de la partie occidentale du bâtiment renferme une grande et remarquable cheminée en pierre, ornée de sculptures et datant de l'époque de Louis XIII. Elle se trouvait dans les deux vastes pièces dites "salles des Francs-Maçons ", avec fresques et cheminées à bas-relief d'époque Louis XIII qui auraient été démolies vers 1825. En 1879, cette pièce servait de cuisine. Mme Borot léguait également à la commune de Vézelay les meubles meublants et objets d'art, indiquant que la bénéficiaire pourrait vendre les meubles qui ne lui seraient d'aucune utilité, à l'exception des tapisseries destinées à garnir l'immeuble ainsi que les objets d'art proprement dits. Conformément au vœu de Mme Borot, qui "priait la commune de bien vouloir, en souvenir de son cher mari, donner le nom de sa famille à une des salles de la propriété ", les deux salles de réception, situées au rez-de-chaussée de la partie orientale du bâtiment actuel, sans doute construites au milieu du XIX ${ }^{\mathrm{e}}$ siècle, ont pris le nom de leur ancien propriétaire.

Figure 5

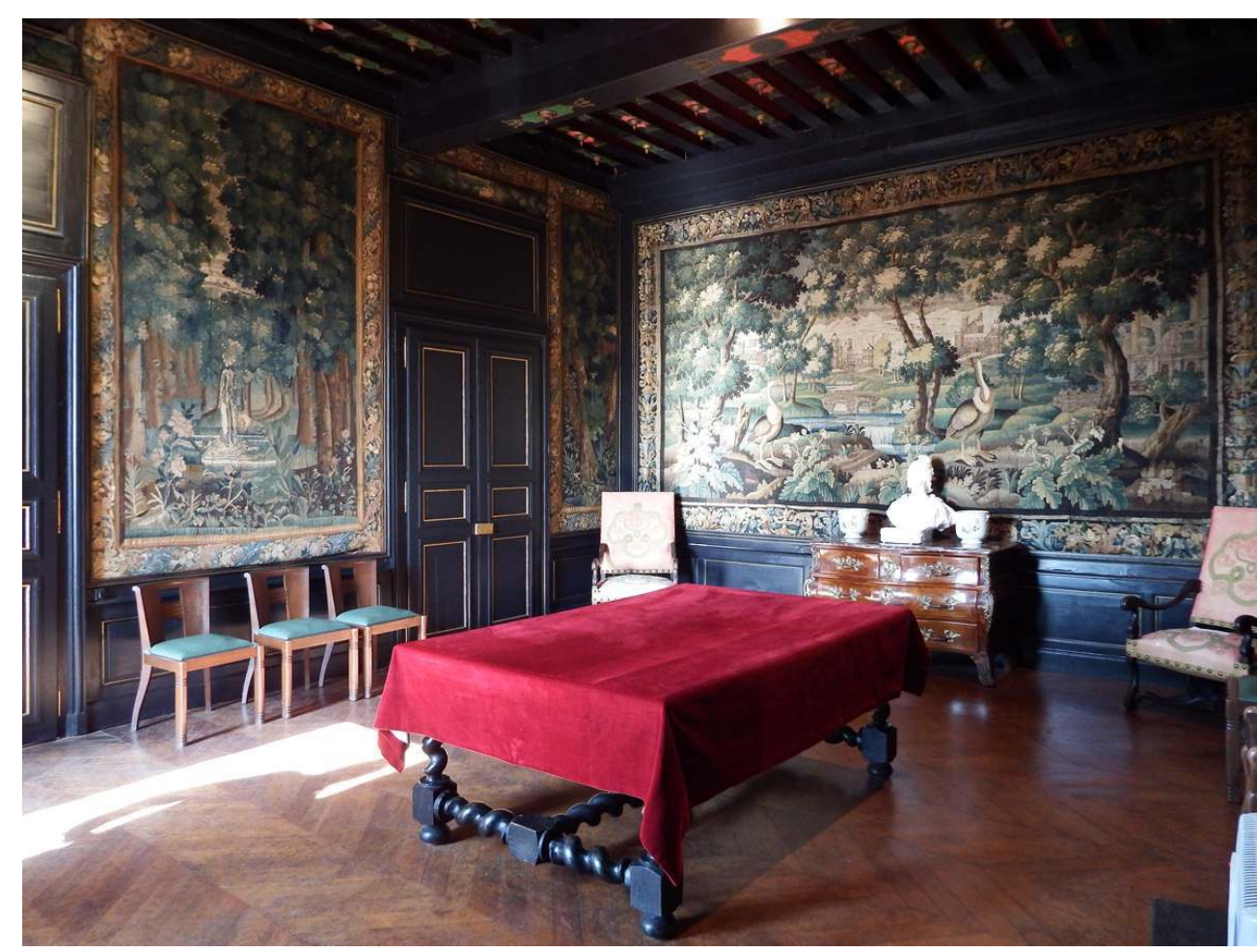

Vue d'ensemble des salles Borot, Vézelay, Mairie.

(c) CAOA de I'Yonne. 
Figure 6

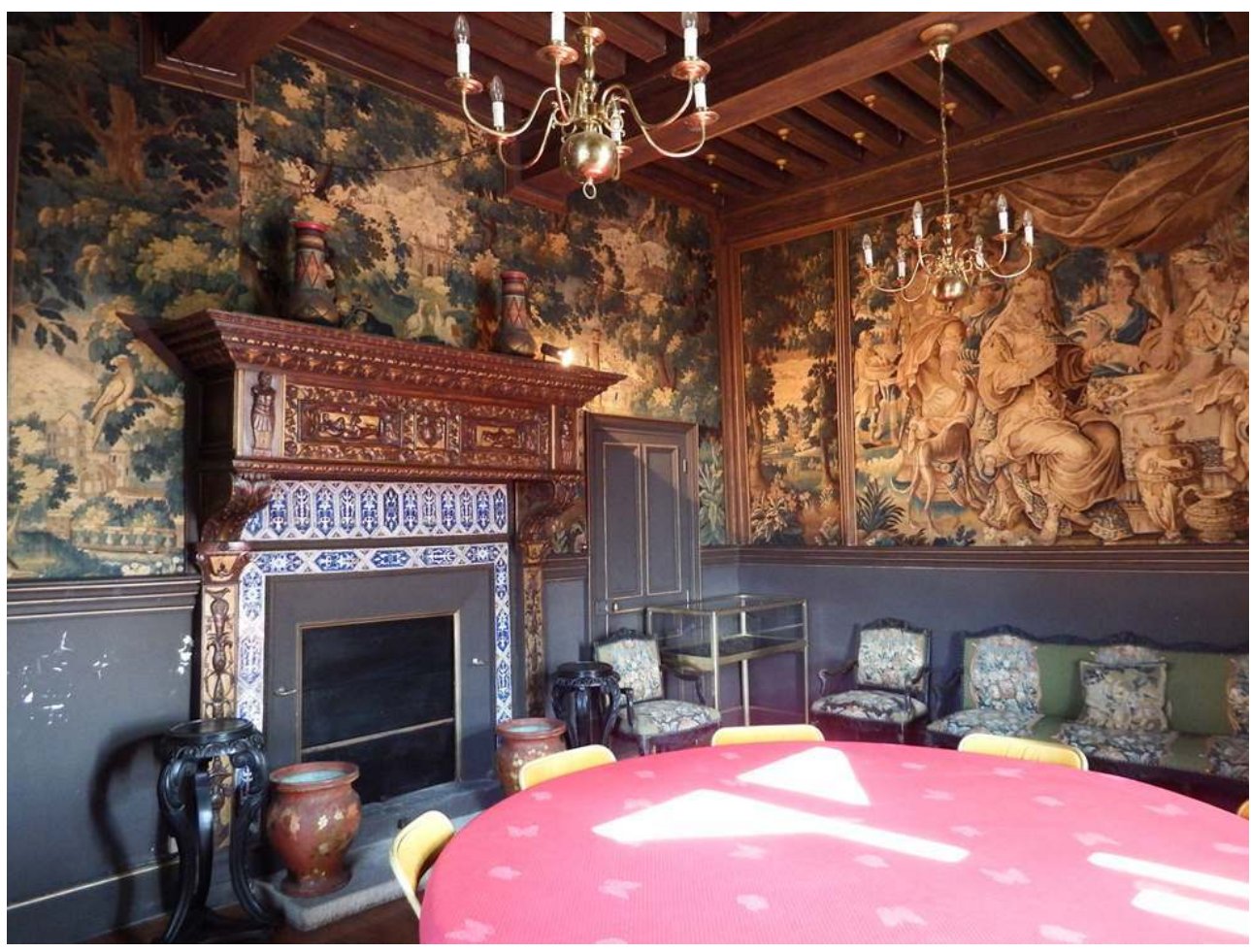

Vue d'ensemble des salles Borot, Vézelay, Mairie.

(c) CAOA de I'Yonne.

11 Les deux "salles Borot» (fig. 5, fig. 6) sont garnies sur la totalité de leurs murs de tapisseries placées dans des boiseries peintes en noir. Il s'agit de verdures ou sous-bois fleuris au milieu desquels se dressent des châteaux, des ruines, des ponts parmi lesquels évoluent des oiseaux (fig. 7). Les tapisseries de ces salles ont été transformées, coupées et réadaptées lorsqu'elles furent installées à Vézelay, au milieu du xIX ${ }^{e}$ siècle mais depuis, rien n'a bougé. Deux scènes figurées complètent cet ensemble: une scène de la vie d'Alexandre et une scène de la vie seigneuriale. Une autre tapisserie de verdure encadrée est accrochée dans le grand escalier montant du rez-de-chaussée au premier étage. Enfin, une banquette trois places, deux chaises, et trois fauteuils sont recouverts de tapisseries ornées de pavots et réalisées spécialement pour ces sièges. Complétant l'ensemble, deux chaises, conservées dans la bibliothèque, sont tendues de fragments de grandes tapisseries récupérés dans des chutes. Toutes ces tapisseries, tentures ou garnitures de sièges, proviennent soit des ateliers d'Aubusson des XVII et XVIII ${ }^{e}$ siècles, soit d'ateliers flamands des $\mathrm{XVI}^{\mathrm{e}}$ et $\mathrm{XVII}^{\mathrm{e}}$ siècles. Elles ont été classées au titre des monuments historiques par arrêté du 23 octobre $1991^{10}$. 
Figure 7

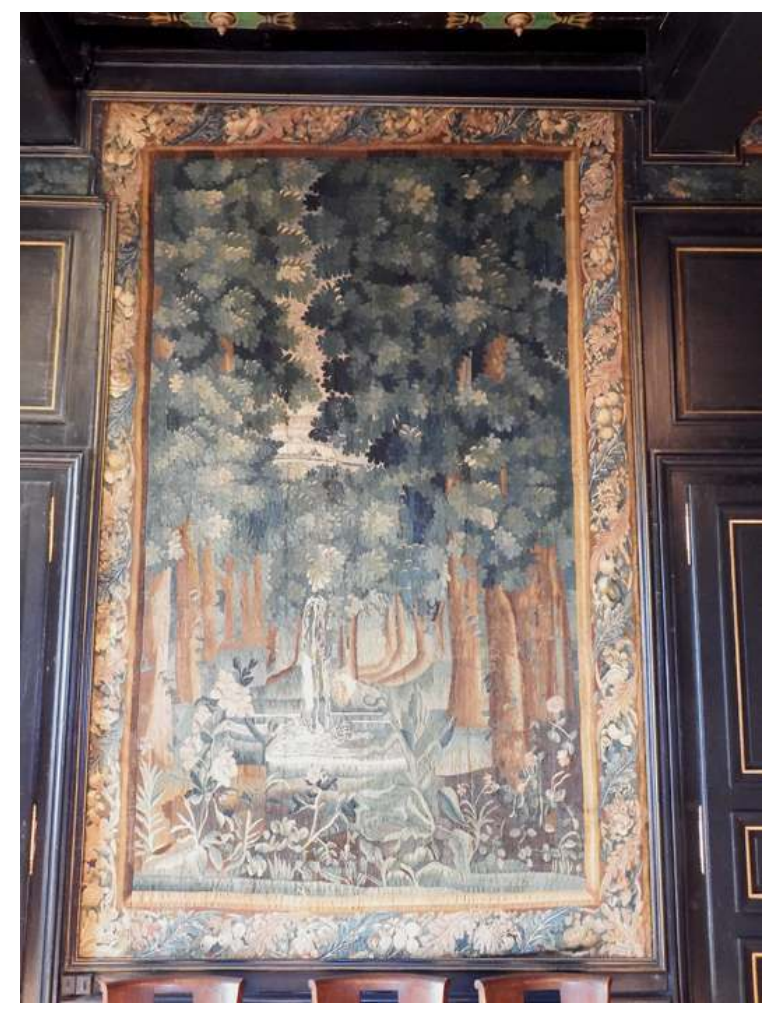

Tapisserie de l'une des salles Borot, Vézelay, Mairie.

(c) CAOA de I'Yonne.

D'autres objets d'art ont été inventoriés et mériteraient d'être également protégés au titre des monuments historiques. Bon nombre étaient dans la maison au moment du legs. Certains sont mentionnés par Mme Borot dans ses testament et codicilles et l'ensemble a été inventorié et estimé en 1925. Si le testament, qui a conduit à l'installation de la mairie de Vézelay dans un hôtel particulier, a permis de conserver l'ensemble des collections in situ, le récolement régulier apparaît toutefois nécessaire ; certains fauteuils ont changé de pièce au gré des activités communales, ou même de bâtiment, ce qui en rend la localisation délicate.

13 Ces ensembles restent fragiles, leur importance historique et artistique étant fréquemment oubliée par nos contemporains. Tel est le cas des théâtres qui, lors de restaurations, perdent une grande partie de leur mobilier et de leur décor. Les protections du mobilier du théâtre de Dijon ou du théâtre d'Auxerre illustrent la considération récente dont ces ensembles font l'objet ${ }^{11}$.

\section{La redécouverte des lieux publics, l'exemple des théâtres}

14 Les enquêtes menées dans les années 1970-1980 sur le xix siècle, ainsi que celle du service régional de l'Inventaire sur les théâtres dans les années $1990^{12}$, ont permis de protéger au titre des monuments historiques les édifices les mieux conservés et les plus intéressants pour leur architecture ou leur décor. Ce sont ainsi dix théâtres qui ont été 
protégés en Bourgogne ${ }^{13}$. Il convient toutefois de noter que peu d'objets mobiliers liés à ces édifices ont fait l'objet de protections.

15 En effet, si les théâtres ont intéressé la conservation régionale des Monuments historiques pour leurs façades, leurs distributions ou leurs décors intérieurs, le mobilier n'a été que rarement pris en compte. Les archives documentent néanmoins très souvent les commandes de sièges pour les foyers ou de divers objets meublants. Aussi, les conservations départementales des antiquités et objets d'art de la région se sont-elles intéressées récemment à ce patrimoine. Si les disparitions sont nombreuses, liées à la modernisation des salles et au besoin de confort des spectateurs, voire au changement de destination de certains espaces, quelques éléments de mobilier ont retenu l'attention et ont été protégés ou vont l'être.

Tel est le cas du théâtre de Dijon, construit de 1810 à 1828 sur l'ancien cloître de la SainteChapelle de Dijon d'après les plans de Jacques Cellerier (1742-1814). Le foyer est, avec la salle de spectacle, au centre de toutes les attentions. Le Monde dramatique le note d'ailleurs en 1837 en indiquant que l'ensemble «en vaut la peine, plus qu'on ne le saurait croire peut-être $^{14} »$. Il se présente aujourd'hui encore avec ses colonnes corinthiennes, ses cheminées de marbre surmontées de miroirs et ses fenêtres ouvrant sur la place du théâtre. Afin de le meubler, la Ville de Dijon commanda à Jean-Jacques Werner (1791-1849), tapissier du roi, 25 rue du Bouloi à Paris ${ }^{15}$, un ensemble de meubles qui a miraculeusement survécu aux rénovations successives et a été inscrit au titre des monuments historiques en 2013 (fig. 8). Cet ameublement en frêne et velours rouge à l'origine (bleu aujourd'hui), se compose de 12 fauteuils, 12 chaises à barrettes, 6 chaises à dossiers pleins et 8 banquettes de tailles diverses. L'ensemble est typique du style Charles X qui affectionne les bois clairs. Les meubles ont été livrés en 1828 par la maison parisienne, en même temps que les lustres et les rideaux. 


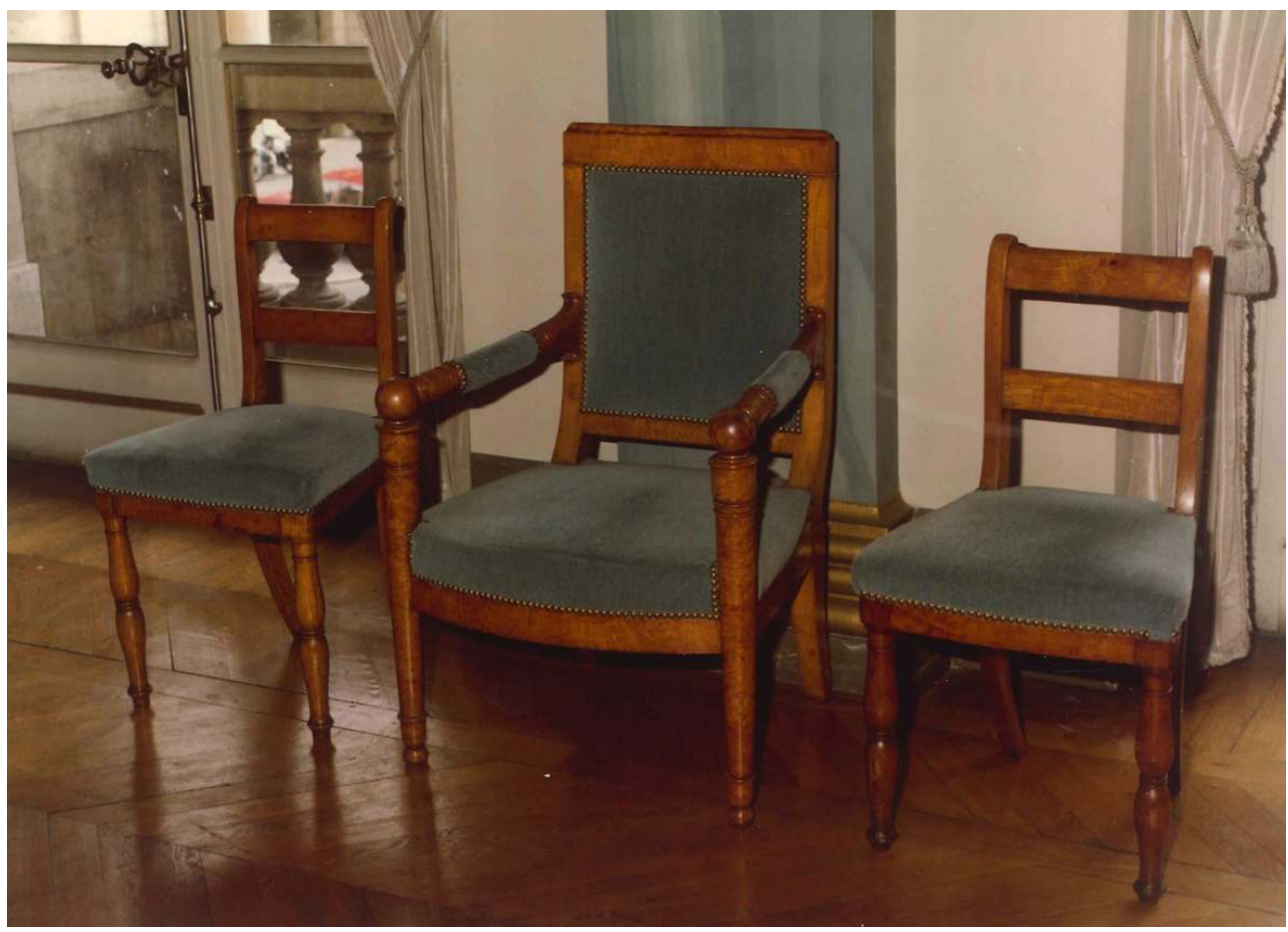

Jean-Jacques Werner, Un fauteuil et deux chaises, 1828, Dijon, Théâtre municipal.

(c) CAOA de Côte-d'Or.

17 La protection de cet ensemble se justifie à la fois par les liens entre le mobilier et les lieux qui l'abritent, les ensembles de chaises et de fauteuils venant se positionner sur certaines boiseries, mais également en raison du renom de l'ébéniste et de la documentation abondante fournie par les archives à propos de cette commande. La difficulté majeure réside dans la gestion quotidienne de cet ensemble, le foyer étant régulièrement utilisé comme salle de concert ou de répétition. Lors de l'inventaire, une partie du mobilier fut d'ailleurs retrouvé dans les caves et dans divers bureaux. Le travail de protection devrait permettre de sensibiliser les utilisateurs à ce mobilier.

Un ensemble bien différent a été récemment redécouvert en Bourgogne : créé presqu'un siècle plus tard, commencé en 1937 dans le style Art déco, le théâtre d'Auxerre, conçu comme "maison du peuple », n'a ouvert ses portes qu'en 1950, le chantier étant freiné par la guerre. Le décor intérieur est commandé à Jean Burkhalter (1895-1982), peintre décorateur originaire d'Auxerre. Outre les peintures des murs et du plafond de la salle de spectacle, qu'il réalise entre 1939 et 1942, l'artiste a conçu deux panneaux exécutés par la manufacture de Sèvres. Il collabore en effet quelques années avec la manufacture qui présente les deux panneaux dans son pavillon à l'Exposition universelle de 1937. L'Ouie ( fig. 9) et La Vue étaient accompagnés d'un plafond. Seuls les deux panneaux purent être achetés par la Ville d'Auxerre pour son nouveau théâtre. Plusieurs documents conservés à la manufacture de Sèvres ou dans les collections du musée d'Auxerre en témoignent. 


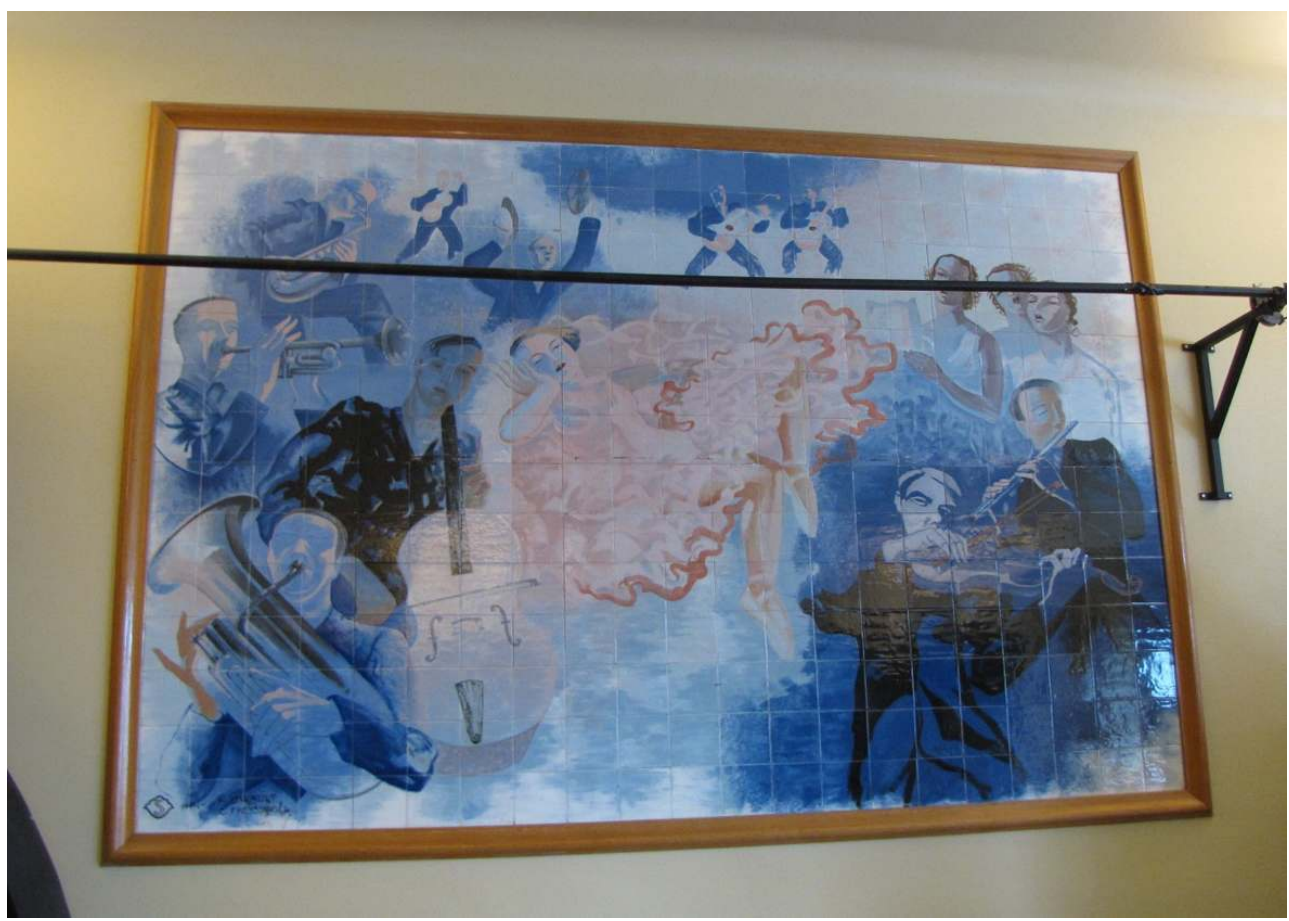

Manufacture de Sèvres, d'après Jean Burkhalter, L'Ouie, 1937, Auxerre, Théâtre municipal.

Phot. Vottero, Michaël. (c) DRAC Bourgogne Franche-Comté.

19 L'inscription du théâtre en 2012, dans le cadre de l'enquête conduite par la conservation régionale des Monuments historiques sur le patrimoine $\mathrm{du} \mathrm{xx}^{\mathrm{e}}$ siècle, a permis de redécouvrir ces deux œuvres et d'en proposer la protection lors d'une future commission départementale des objets mobiliers.

Si le théâtre d'Auxerre apparaît comme un édifice qui a perdu l'ensemble de son mobilier d'origine, la présence de ces deux éléments réalisés par un enfant du pays souligne la prise en compte du patrimoine $\mathrm{du} \mathrm{xx}^{\mathrm{e}}$ siècle. Patrimoine fragile, le mobilier des théâtres n'en demeure pas moins un élément essentiel, au même titre que les plafonds peints qui décorent les salles ou les foyers. Complément indispensable, il a souvent eu tendance à disparaître au fil des ans pour libérer de l'espace, moderniser les lieux ou tout simplement par changement de goût.

21 Il en est de même des vastes collections hospitalières qui, délaissées par la modernisation des lieux, sombrent bien souvent dans l'oubli et sont menacées par les déménagements réguliers des structures de leurs sites d'origine.

\section{Les ensembles hospitaliers, des collections publiques ou privées ?}

Nevers, Dijon, Paray-le-Monial, Chalon-sur-Saône... les hôpitaux de Bourgogne, comme partout ailleurs, sont touchés par des déménagements, certains centres hospitaliers quittant pour la première fois leur site historique, à l'image du centre hospitalier de Dijon ${ }^{16}$. L'interrogation des bases de données nationales laisse deviner l'importance de leurs collections. Une veille accrue doit ainsi être mise en place lors du transfert de services ou 
d'établissements. Les travaux sont en effet sources de disparitions ou de dégradations, comme cela s'est produit pour l'horloge de la chapelle de l'hôpital de Nevers, volée lors de la transformation de l'hôpital en logements et centre commercial. Lorsque l'on sait que l'apothicairerie de l'hôpital d'Alise-Sainte-Reine (Côte-d'Or) renferme 1108 objets ( fig. 10, fig. 11), on comprend l'importance d'une veille régulière sur ces ensembles.

Figure 10

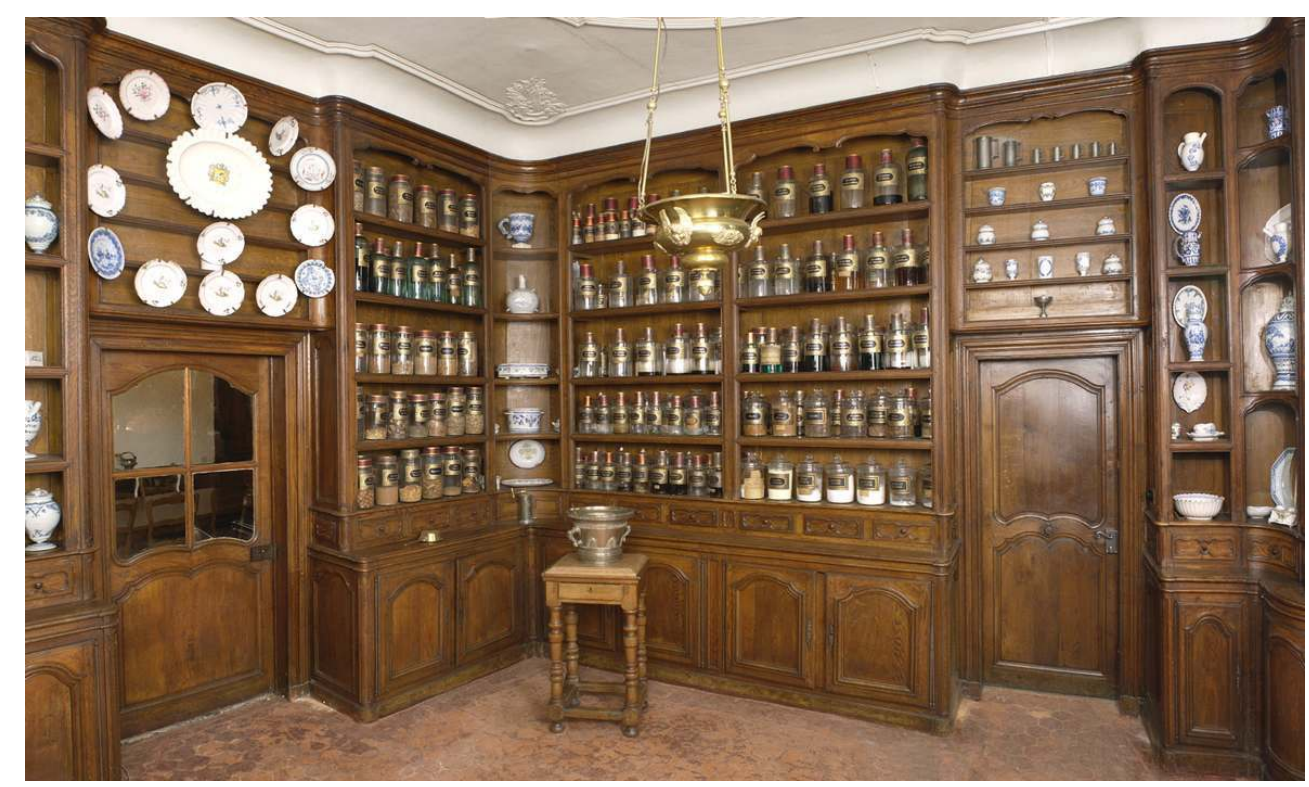

Apothicairerie, Alise-Sainte-Reine, hôpital.

(c) Service Régional de l'Inventaire de Bourgogne-Franche-Comté. 


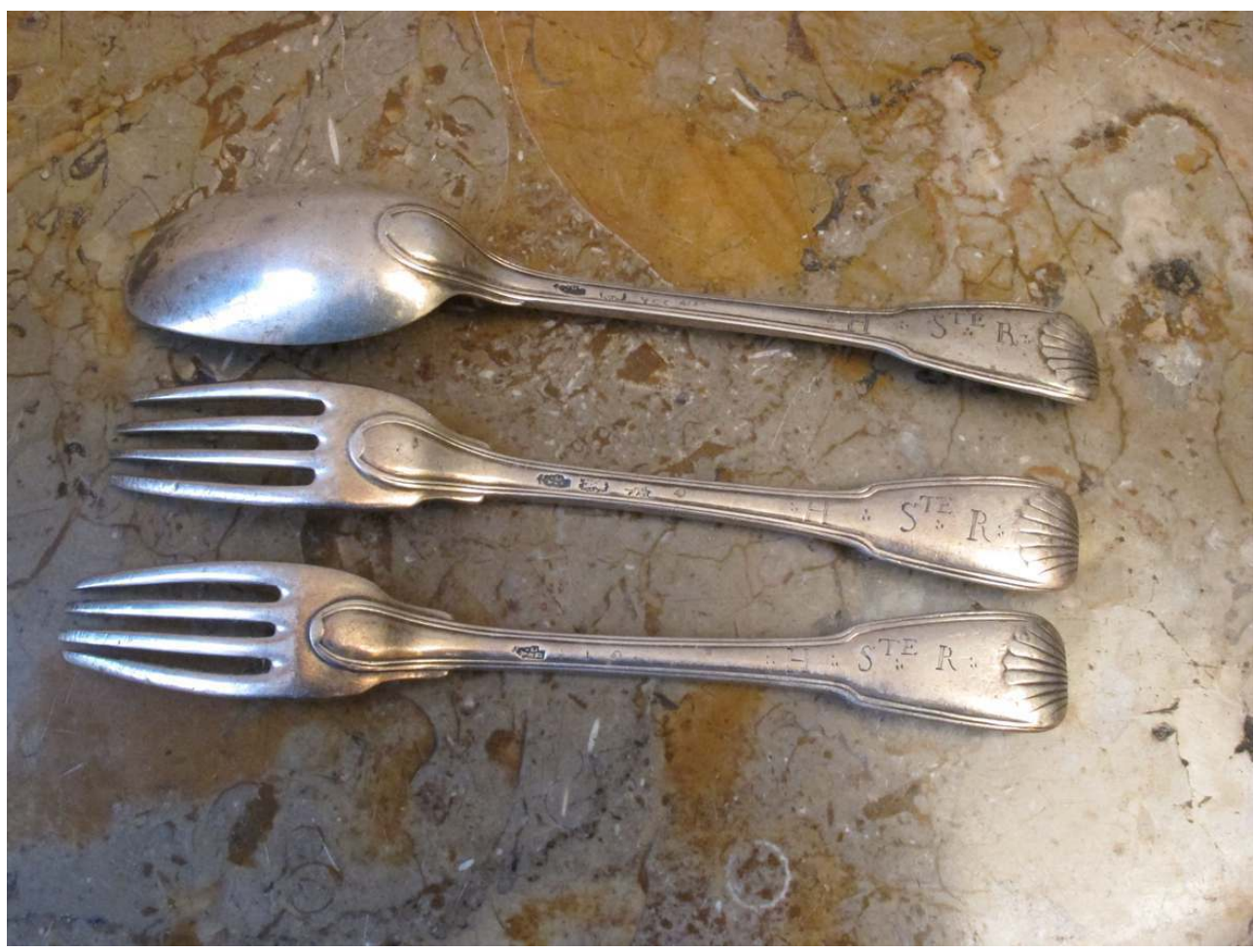

Fourchettes et Cuiller, 1754-1754, Alise-Sainte-Reine, hôpital.

Phot. François, Bruno, 2012. (c) Bruno François.

C'est pour cette raison que l'Agence Régionale de Santé a créé en 1998 un poste de chargé de mission pour le patrimoine des hôpitaux. Bruno François, désormais assisté d'Adeline Rivière, veille à la bonne gestion des collections protégées au titre des monuments historiques en réalisant des inventaires et en proposant de nouvelles protections. Tel est le cas d'un récent rein artificiel des années 1950 de l'hôpital de Dijon, inscrit au titre des monuments historiques le 16 décembre 2013 (fig. 12), ou d'un ensemble de lits du XIX siècle de l'hôpital de Nolay (Côte-d'Or), remisés dans les greniers de cette actuelle maison de retraite, désormais inscrits (fig. 13). Lorsque l'on aborde ce patrimoine, la question du statut de ces collections se pose. 
Figure 12

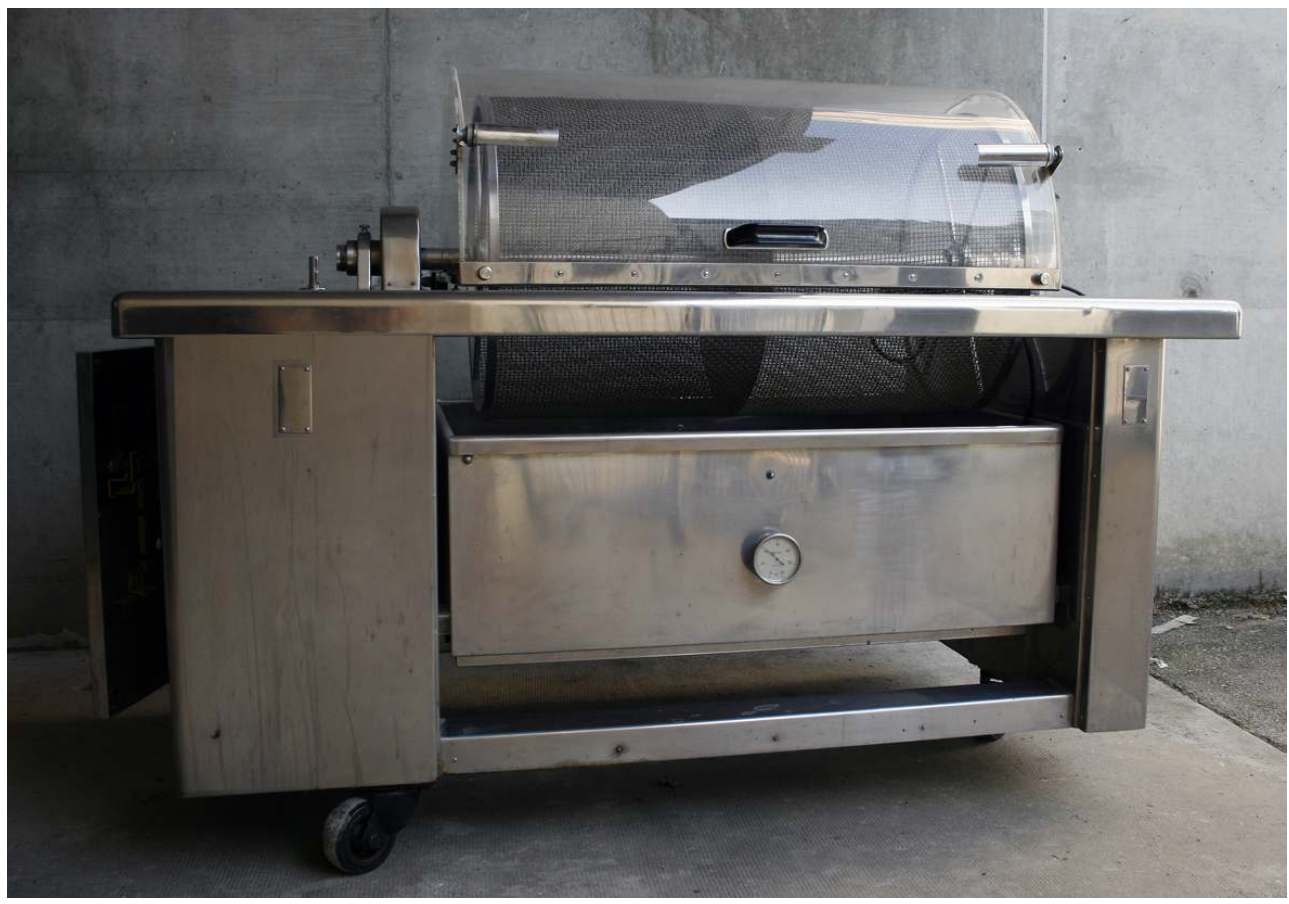

Rein artificiel de marque Necker, milieu des années 1950, Dijon, CHU.

Phot. Ramot, Jean-Pierre. (c) CHU Dijon Bourgogne.

Figure 13

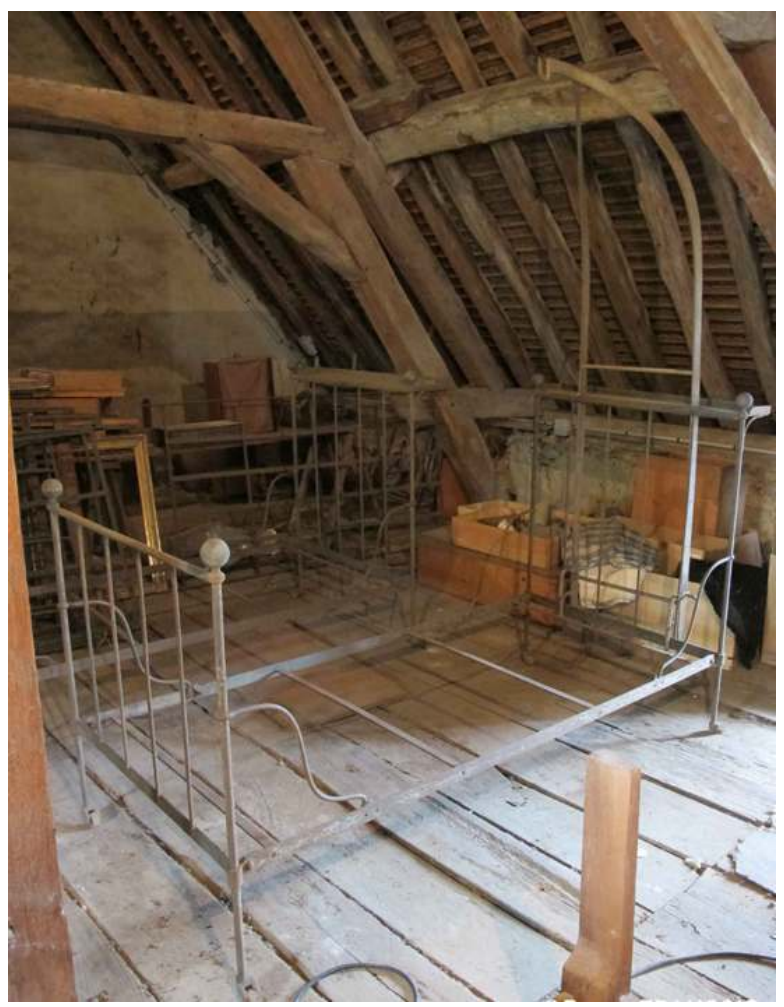

Lit métallique, XIXe siècle, Nolay, EHPAD Jeanne Pierrette Carnot de Nolay.

Phot. François, Bruno. (C) Bruno François - ARS Bourgogne Franche-Comté. 
question est simple, la réponse est complexe car les hôpitaux publics possèdent du patrimoine immobilier ou mobilier qui peut relever du domaine public ou privé ${ }^{17}$. Comme le confère l'article L. 2111-1 du Code général de la propriété des personnes publiques (CG3P), font partie du domaine public les biens appartenant à une personne publique et qui sont soit affectés à l'usage direct du public, soit affectés à un service public. Dans le premier cas, on parle de bâtiments ou locaux permettant de remplir la ou les missions spécifiques de l'établissement public, c'est-à-dire les soins pour les établissements de santé. Ainsi, les apothicaireries et leurs collections de pots peuvent être considérées comme relevant du domaine public, même lorsqu'elles ne sont plus en service depuis plusieurs décennies. Dans le second cas, la domanialité publique des bâtiments est déterminée par la nature du service public rendu, sans leur présence, le service public ne pourrait avoir lieu. Par ailleurs, le CG3P indique également que relèvent du domaine public les objets présentant un intérêt public du point de vue de l'histoire de l'art, de l'archéologie, de la science ou de la technique (article L. 2112-1). Cette définition est principalement issue de l'article 14 de la loi du 31 décembre 1913 relative aux monuments historiques. En dehors des objets protégés au titre des monuments historiques il est souvent compliqué, aux yeux des établissements, de définir ce qui présente un intérêt du point de vue de l'art ou des sciences et techniques. Pour prendre des exemples culturels dans les hôpitaux, on citera le cas des livres dans les bibliothèques, des œuvres présentées dans les services, cours et jardins avec une signalisation spécifique. Même les objets de soins réformés ${ }^{18}$, présentés dans le cadre de parcours muséographiques aménagés spécifiquement pour le public, peuvent être considérés comme faisant partie du domaine public de l'établissement public et ce, même s'il s'agit d'un service public secondaire pour les établissements de soins. Ce dernier exemple montre combien la frontière entre domaines public et privé, est souvent bien compliquée à définir et en particulier pour ce qui relève des objets.

Enfin, selon l'article L. 2141-1 du CG3P, lorsqu'un bien n'est plus affecté à un service public ou à l'usage direct du public, il ne relève plus du domaine public à compter de l'intervention de l'acte administratif constatant son déclassement. Ainsi la sortie d'un bien du domaine public hospitalier est conditionnée, d'une part, par une désaffectation matérielle de l'objet et, d'autre part, par une décision du directeur d'établissement la constatant et portant déclassement du bien.

En ce qui concerne les collections patrimoniales des établissements hospitaliers (hors legs ou donation) : objets d'art, d'histoire, scientifiques et techniques, de soins, rares sont ceux ayant bénéficié d'un déclassement. Pourtant, la gestion, et en particulier les ventes dont ils font bien souvent l'objet, laissent à penser qu'ils sont considérés, à tort, par les chefs d'établissement comme relevant du domaine privé de l'établissement public. Ce statut juridique complexe et le désintérêt vis-à-vis du patrimoine de structures dont la mission principale est le soin des patients rendent aléatoire la conservation du mobilier. Seules les protections au titre des Monuments historiques semblent pouvoir aujourd'hui conférer un statut et une reconnaissance aux objets suffisamment prégnants pour motiver, si ce n'est de l'intérêt, une gestion minimale. 


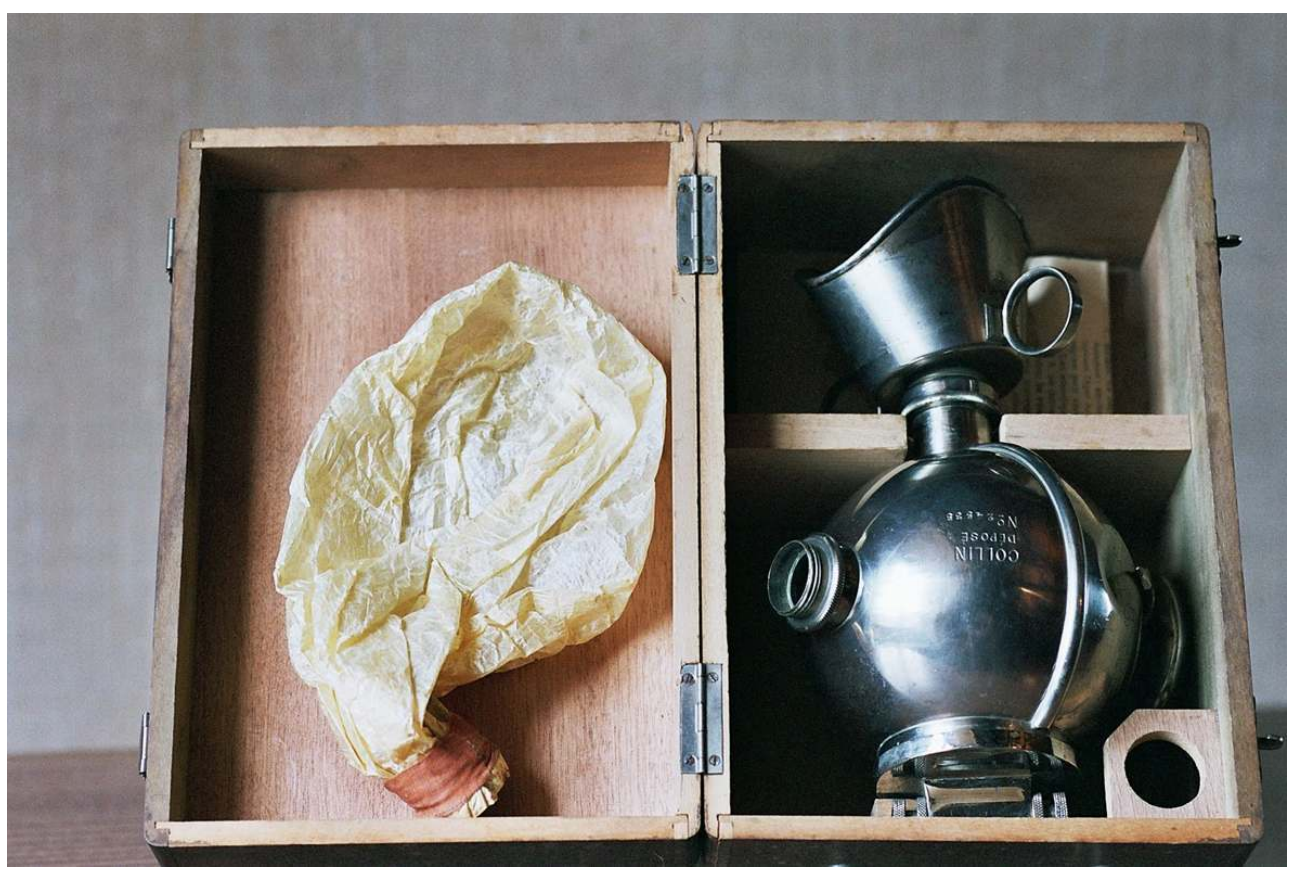

Appareil à éther du professeur Ombrédanne, Avallon, hôpital.

(c) CAOA de I'Yonne.

Ce sont ainsi des inventaires et des protections régulières qui sont établis par les quatre conservations des antiquités et objets d'art de la région. Citons parmi les protections les plus récentes, l'hôpital d'Avallon, où un appareil à éther du professeur Ombrédanne et son coffret ont été inscrits le 10 mai $2012^{19}$ (fig. 14), et l'hôpital de Chalon-sur-Saône ; par ailleurs l'ensemble des cuivres, de l'orfèvrerie et d'une partie du mobilier des sœurs hospitalières de cette institution sera prochainement classé. L'intérêt de ces ensembles d'objets mobiliers est celui d'un témoignage de la vie de ces structures (personnel soignant et patients) mais également de l'évolution des techniques scientifiques et de soin. On trouve ainsi, aux côtés des ustensiles de cuisine, des couverts d'argent, de la vaisselle d'étain portant les initiales de l'hôpital... (fig. 15) Dans le domaine des pharmacies et apothicaireries, outre les pots de faïence ou de porcelaine, ce sont les mortiers, les pilons, les balances... qui offrent des ensembles cohérents et qui ont souvent été dispersés entre plusieurs services. Les pots à pharmacie de l'hôpital d'Avallon ont ainsi été rassemblés dans un même meuble ${ }^{20}$ (fig. 16), afin de reconstituer la collection. Le récent départ de l'hôpital général de Dijon a également permis de mettre en place une politique d'expositions temporaires dans le hall principal du site François Mitterrand. Des vitrines sécurisées permettent de présenter, par roulement, des collections historiques ( fig. 17). Ces dernières offrent au public et aux patients des ensembles d'objets du passé qui viennent illustrer des propos sur la vie de l'ancien hôpital ou sur l'évolution des techniques médicales. Le départ des établissements hospitaliers de leurs emplacements d'origine est un phénomène que l'on constate depuis plusieurs années pour d'autres administrations. 
Figure 15

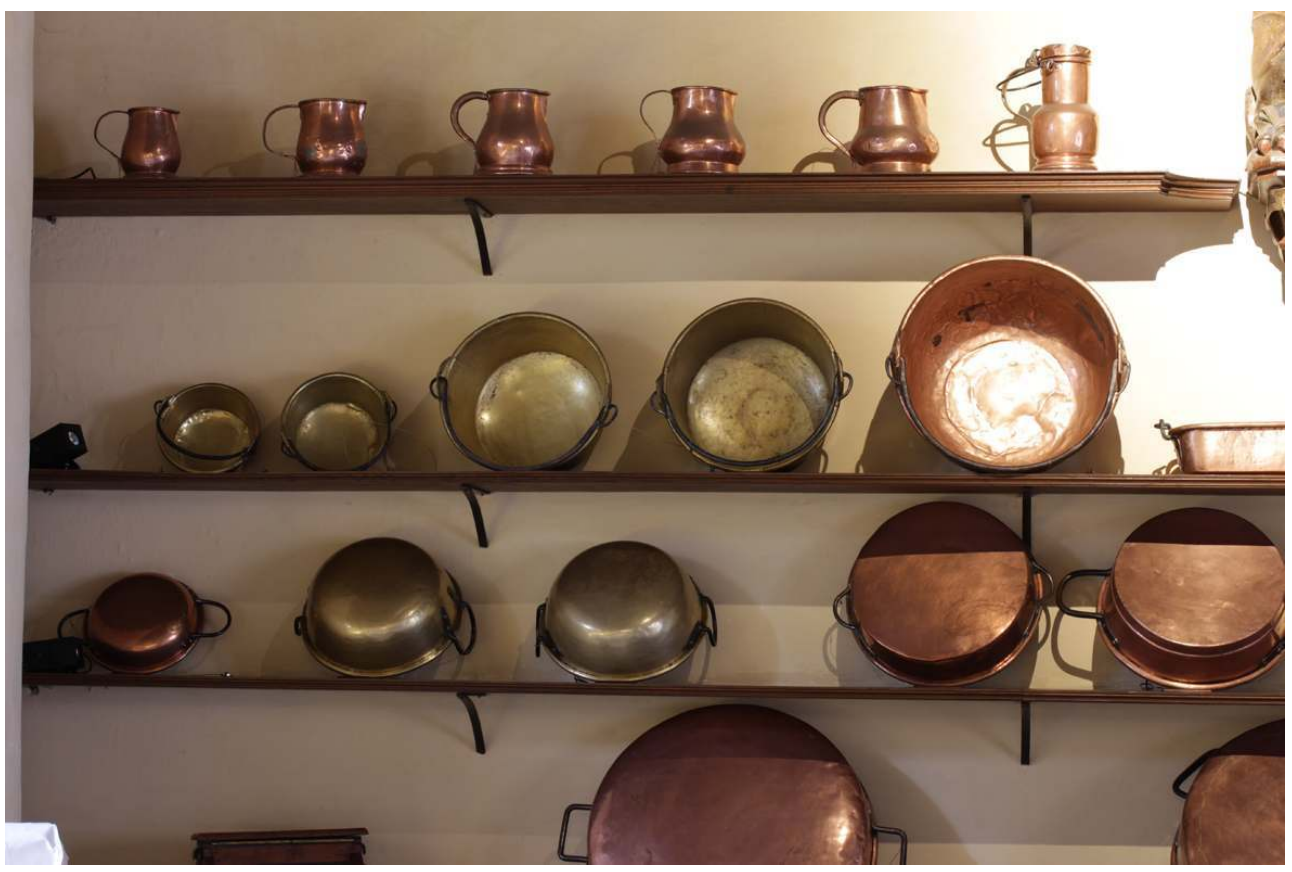

Cuivres de la cuisine des hospices de Beaune.

Phot. François, Bruno. (c) Bruno François - ARS Bourgogne Franche-Comté

\section{Figure 16}

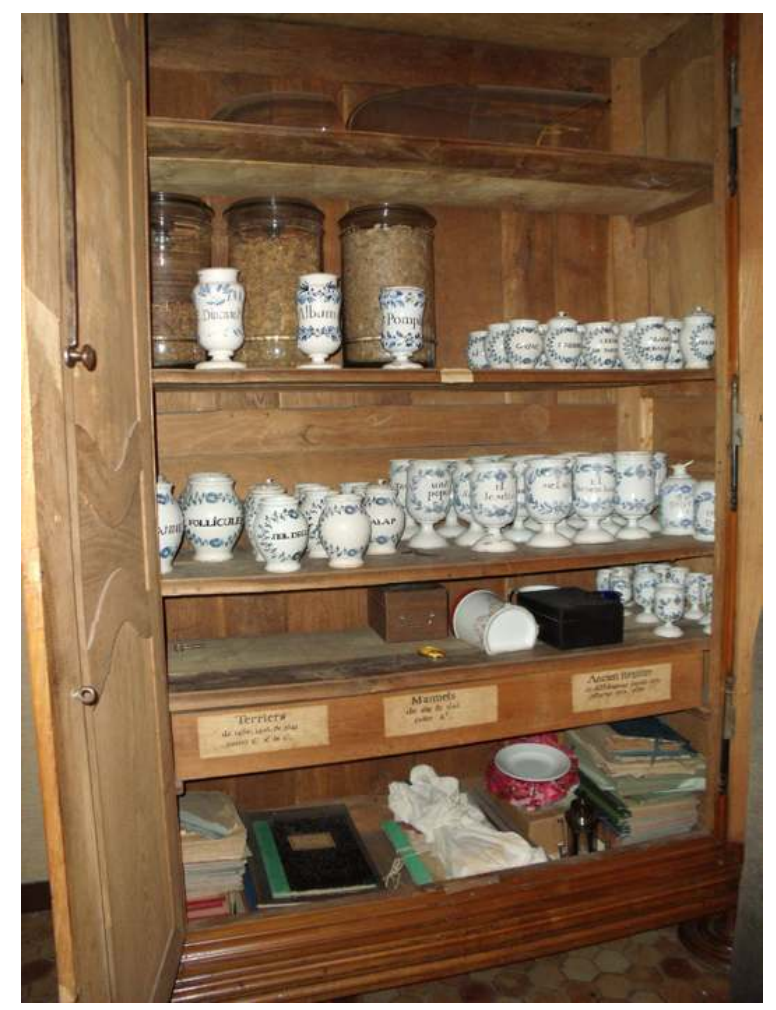

Collection de pots à pharmacie, Avallon, hôpital.

(c) CAOA de I'Yonne. 
Figure 17

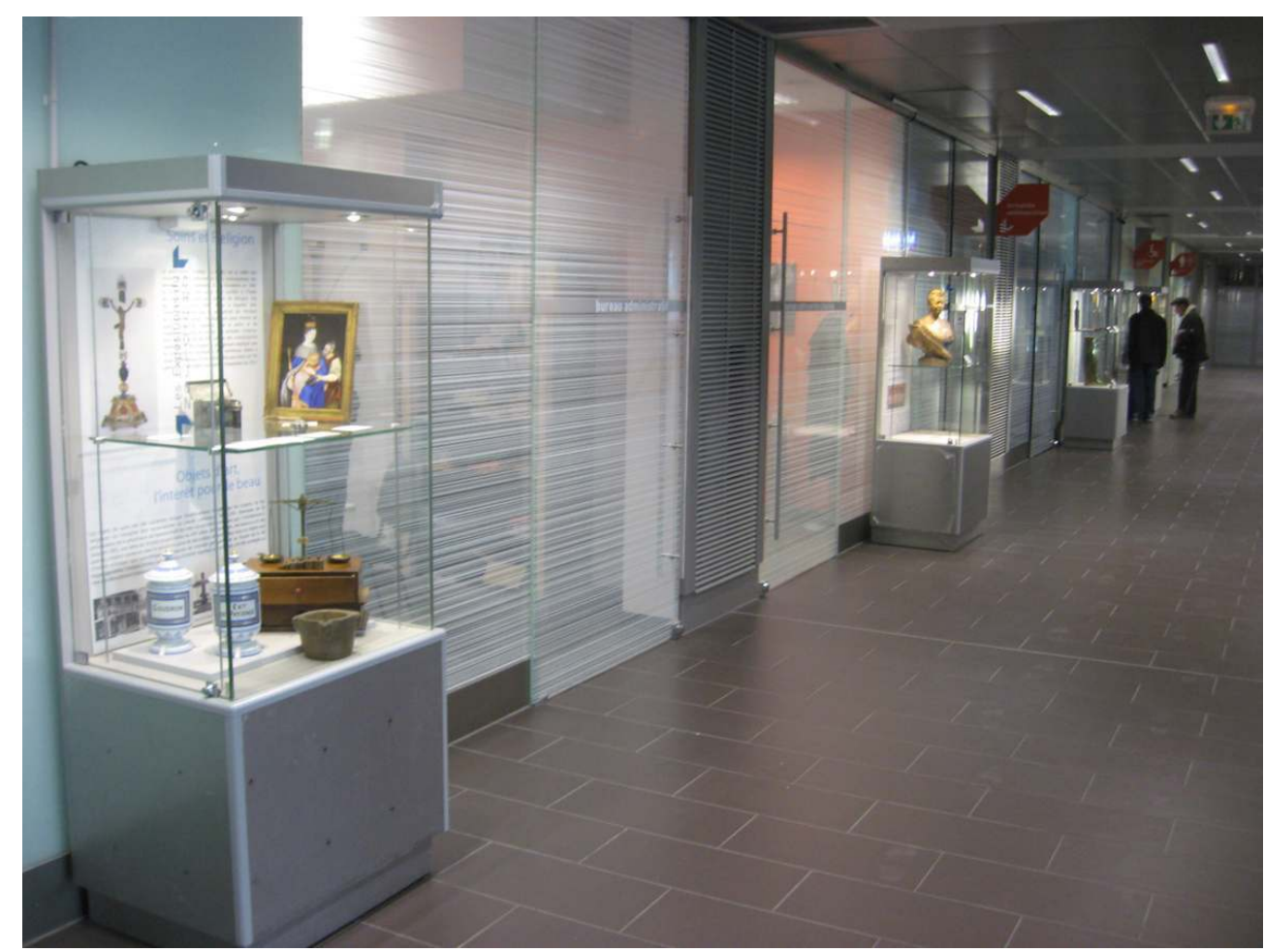

Présentation d'objets mobiliers dans le hall central, Dijon, CHU.

Phot. Vottero, Michaël. (c) DRAC Bourgogne Franche-Comté.

\section{Les administrations bourguignonnes, un nouvel enjeu?}

Les lycées, les universités, les théâtres, les administrations, telles les préfectures et souspréfectures, apparaissent aujourd'hui comme des lieux importants à étudier et à valoriser pour leurs collections. Des campagnes régulières d'étude ont ainsi permis de repérer et de protéger des objets d'art et d'histoire d'intérêt, comme d'un cartel du XVIII ${ }^{\mathrm{e}}$ siècle de l'hôtel de la préfecture de l'Yonne ${ }^{21}$ (fig. 18), inscrit le 10 mai 2012, ou les collections du palais de justice de Dijon. 
Figure 18

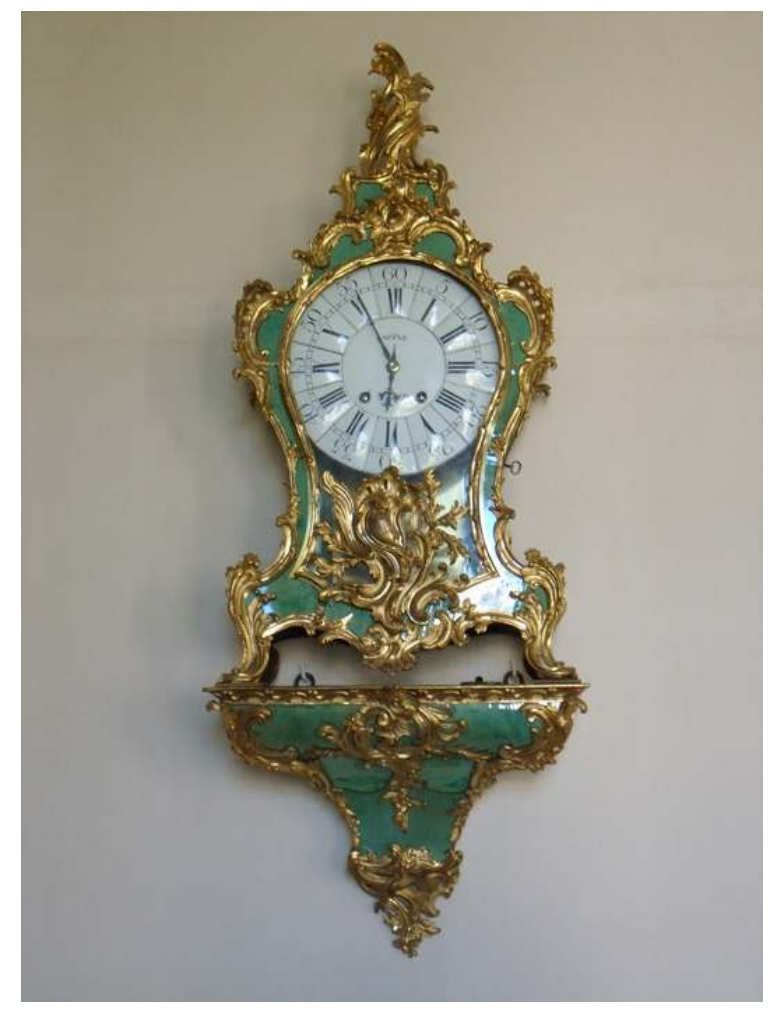

Cartel, XVIIII siècle, Auxerre, Préfecture.

(c) CAOA de I'Yonne.

Dans le cadre du récolement des objets mobiliers protégés au titre des monuments historiques du palais de justice de Dijon, il est apparu qu'un certain nombre de meubles n'étaient pas protégés. La crainte de voir ces ensembles vendus ou démembrés à l'occasion de projets de réaménagement des locaux a motivé un inventaire des collections, aboutissant à la constitution de dossiers pour une partie de ces ensembles, présentés lors de la commission départementale des objets mobiliers de Côte-d'Or du 18 février 2014. La protection d'objets au palais de justice de Dijon n'est toutefois pas nouvelle. Si l'on s'en réfère à la base Palissy, seize objets sont actuellement classés au titre des monuments historiques. Ces derniers sont toutefois, pour la plupart, des objets d'Ancien Régime: plafonds, cartels, fauteuils, cheminées... alors qu'il y a très peu de mobilier du XIX ${ }^{e}$ siècle, paradoxalement très abondant dans cet édifice. Des meubles souvent de série, pour les fauteuils ou les bancs, mais qui témoignent des besoins d'une administration à un moment donné, comme l'image qu'elle se donne de la Justice.

La réforme de la carte judiciaire a entraîné ces dernières années la fermeture de nombreux édifices. Pour la région Bourgogne, certains ont été vidés sans aucune concertation avec les services patrimoniaux locaux (DRAC et CAOA) ou nationaux (ministère de tutelle, MCC), le moindre élément de décor mis en vente. Ces déménagements ont pu être l'occasion de ventes d'objets relevant du domaine public, de la dispersion et de la destruction de décors anciens dans un laps de temps très court. Un phénomène identique à celui de nombreux édifices militaires désaffectés dont les collections ont parfois disparu. 
Pour le palais de justice de Dijon, un certain nombre d'inventaires mobiliers sont conservés aux archives départementales de Côte-d'Or ${ }^{22}$. Les deux ensembles les plus importants, encore en place, résultent de deux commandes en 1841 et 1888. La nouvelle salle d'assises, construite de 1862 à 1869 d'après les plans de l'architecte départemental Charles Suisse (1846-1906), reprend le style Louis XIII pour ses boiseries. La salle intègre le plafond de l'ancienne cour des comptes. Les meubles de cette salle n'ont toutefois pas été réalisés spécifiquement pour cette dernière ; l'ensemble des fauteuils provient en effet d'une commande antérieure. En 1841 et 1842, le mobilier de la chambre dorée fut dessiné par l'architecte Charles Saint-Père (1804-1895). Si les chaises n'ont pas été retrouvées, les fauteuils de la salle des assises correspondent aux dessins conservés de Saint-Père ( fig. 19).

Figure 19

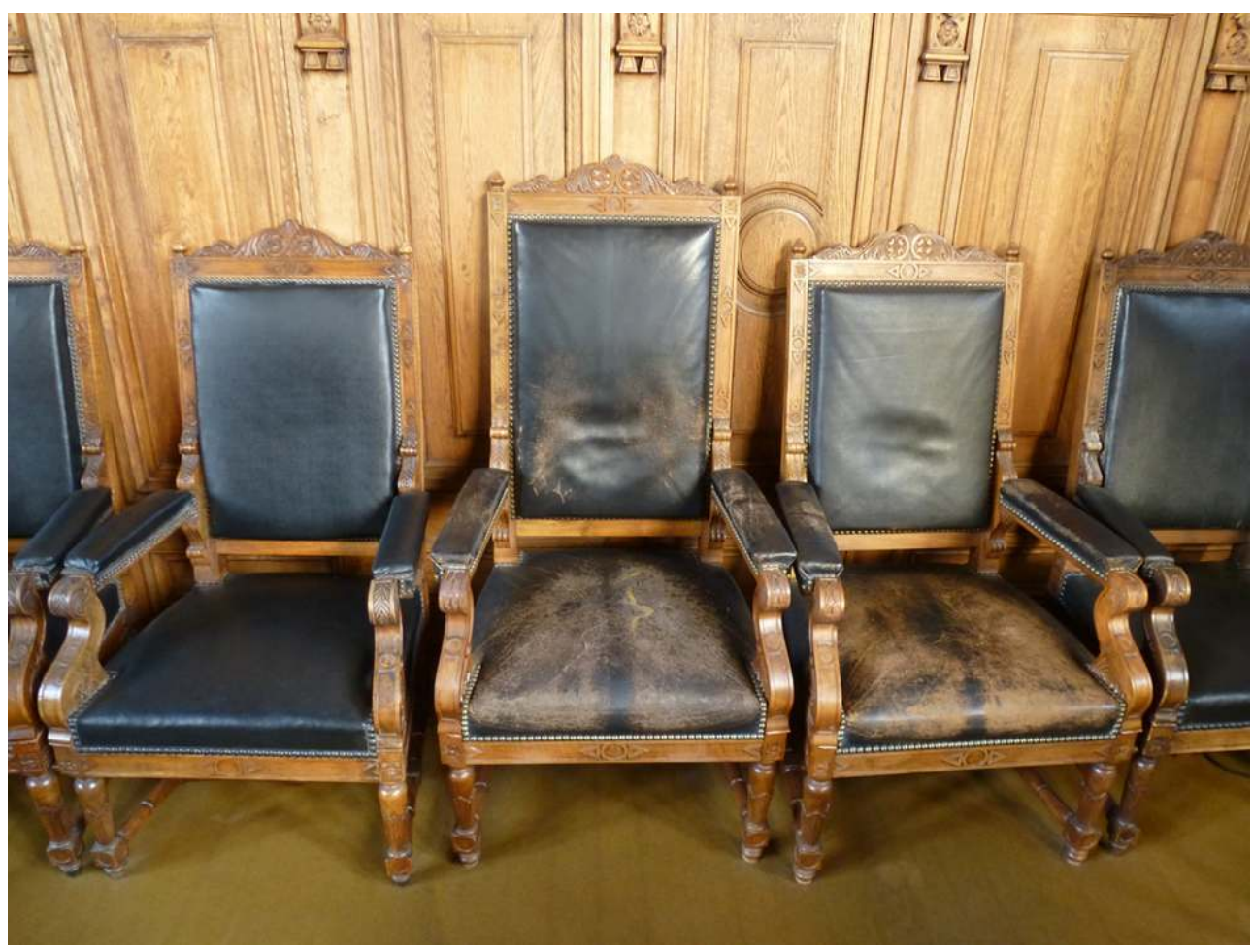

Charles Saint-Père, Ensemble de fauteuils, 1841-1842, Dijon, Palais de Justice.

Phot. Vottero, Michaël. (c) DRAC Bourgogne Franche-Comté.

La chambre dorée, actuelle chambre civile, conserve un ensemble commandé en 1888 ( fig. 20, fig. 21). La protection au titre des monuments historiques d'un certain nombre de meubles a permis de sensibiliser le personnel du palais de justice à une meilleure gestion des collections. Ce mobilier du XIX ${ }^{\mathrm{e}}$ siècle, on le comprend, parfois lourd et peu commode, peut être victime de vastes chantiers de restauration. Il convient néanmoins de veiller à la conservation de ces ensembles non négligeables, de proposer des modes de gestion aux utilisateurs, afin d'éviter des disparitions. Si les collections du palais de justice de Dijon

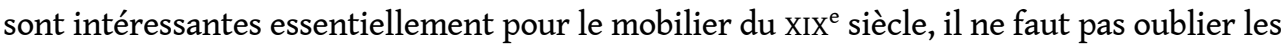
vastes ensembles du $\mathrm{xx}^{\mathrm{e}}$ siècle qui meublent aujourd'hui certains palais de justice. 
Figure 20

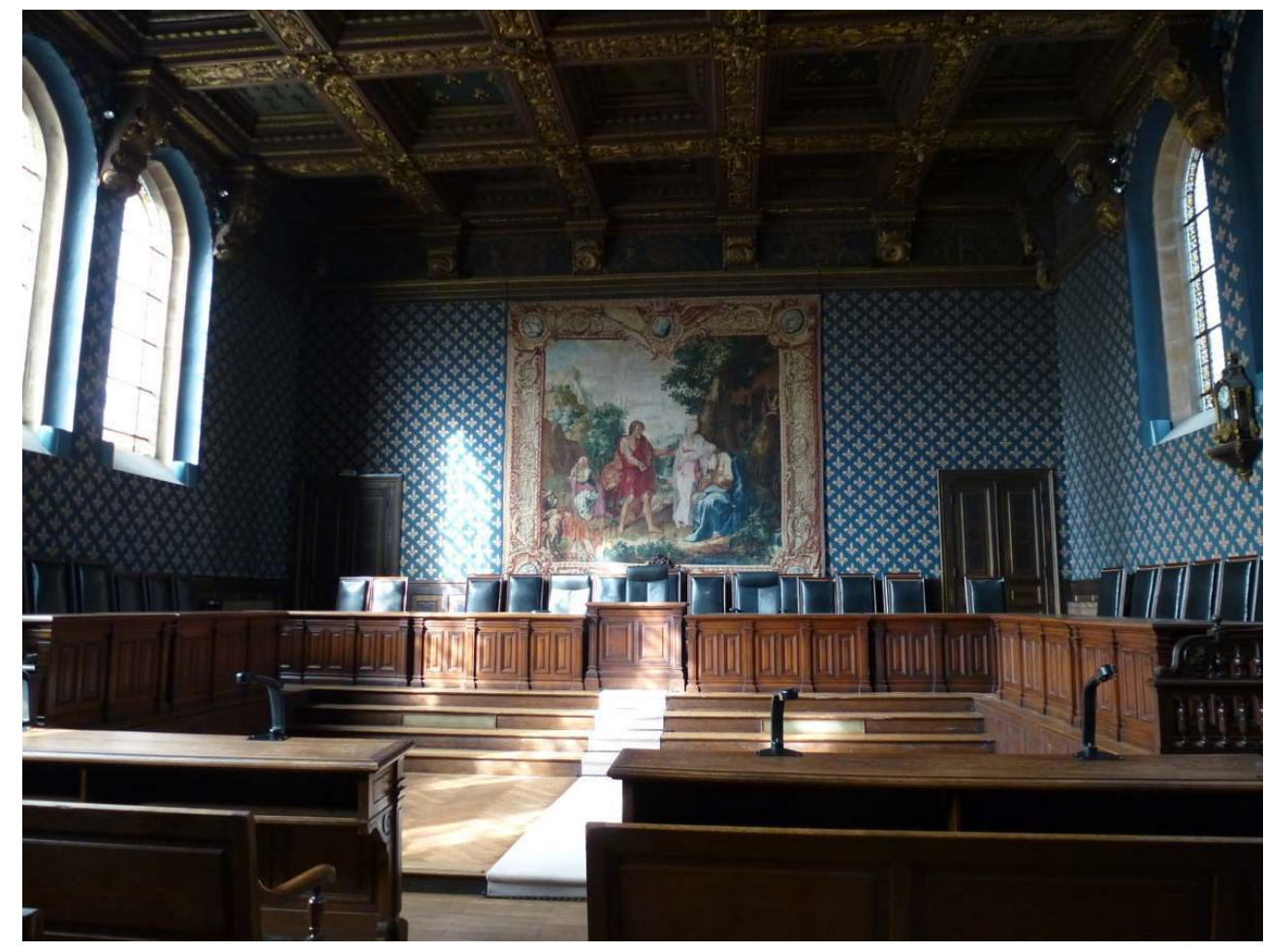

Vue d'ensemble de la salle dorée, Dijon, Palais de Justice.

Phot. Vottero, Michaël. @ DRAC Bourgogne Franche-Comté. 


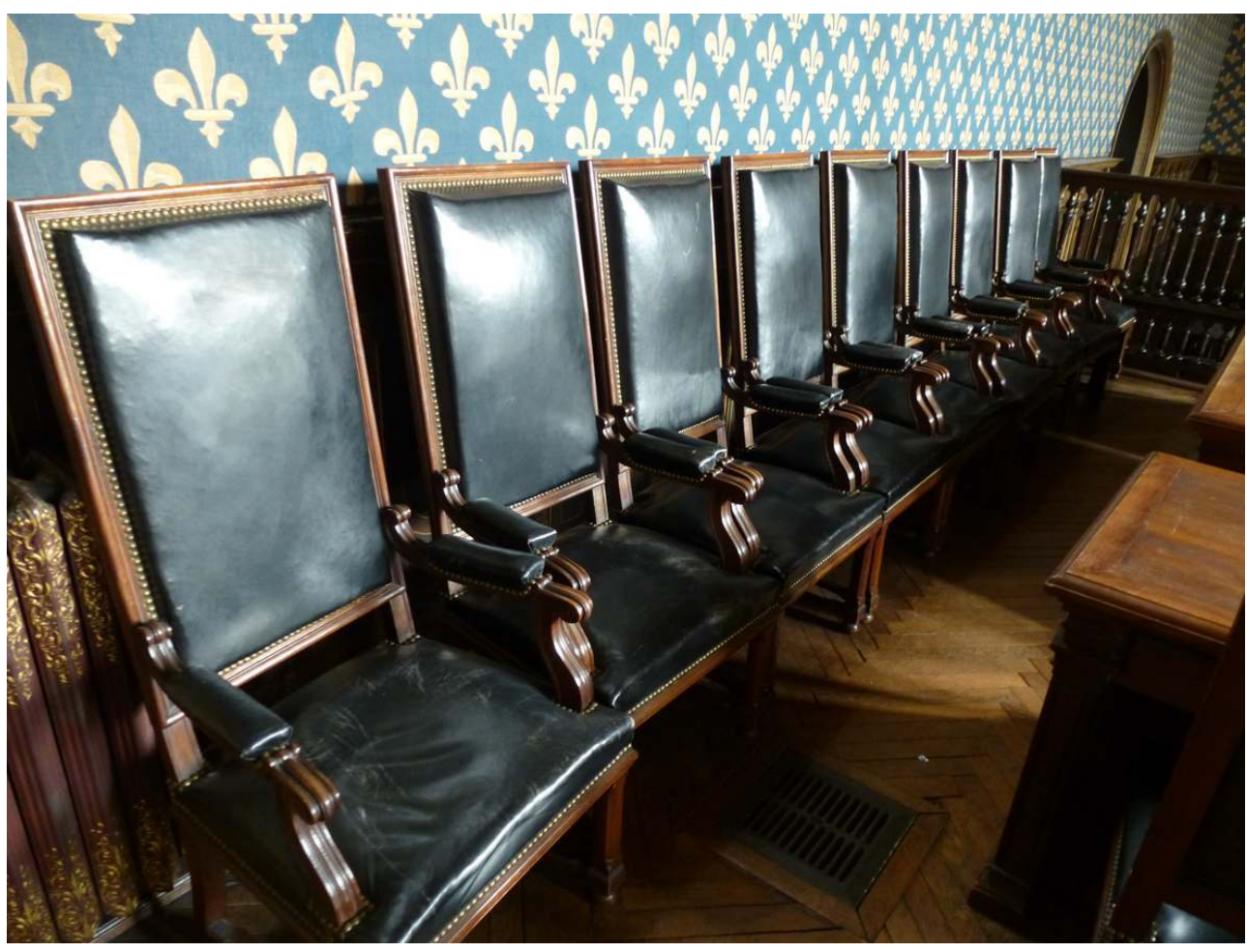

Charles Cotétidor, Ensemble de fauteuils, 1888, Dijon, Palais de Justice.

Phot. Vottero, Michaël. ( ) DRAC Bourgogne Franche-Comté.

On le voit, le patrimoine civil, que l'on étudie et protège depuis quelques années en Bourgogne, reste un vaste domaine de recherche. Ces protections régulières continuent de s'étoffer parallèlement à l'étude du patrimoine religieux déjà bien connu. De nouvelles thématiques civiles sont d'ores et déjà programmées autour des bannières laïques, des collections de lycées ou de préfectures. Ces objets mobiliers variés sont révélateurs d'importants changements politiques et administratifs, de modes de vie ou d'usages passés. Leur intérêt est souvent plus historique qu'artistique mais ils donnent, notamment les objets meublants, un sens aux édifices qui les contiennent et pour lesquels ils ont été spécifiquement achetés ou créés. Des ensembles dont il convient de préserver l'unité car ils sont les témoins de notre histoire.

\section{NOTES}

1. - Nous renvoyons sur le sujet aux articles suivants : KAGAN, Judith. « Des antiquités et objets d'art au patrimoine mobilier». Dans PALOUZIÉ, Hélène (dir.). Icônes et idoles, regards sur l'objet monument historique. Arles: Actes Sud, 2008, p. 23-39, et POISSON, Olivier. «L'objet monument historique ». Ibid., p. 41-52. 
2. - Si les façades sont inscrites depuis 1995 , l'escalier d'honneur avec sa cage, la salle d'angle au rez-de-chaussée, le vestibule du premier étage, la salle du conseil municipal, la salle des mariages et le grand salon d'honneur à l'étage sont classés au titre des monuments historiques par arrêté du 30 mai 1997. Sur l'histoire de l'hôtel de ville de Sens et de ses collections, nous renvoyons à l'ouvrage : Sens, l'hôtel de ville a 100 ans, catalogue de l'exposition, Sens, Orangerie des musées de Sens, 12 décembre 2004-13 mars 2005. Sens : musées de Sens, 2004.

3. - Le patrimoine des hôpitaux de Bourgogne a été largement étudié par le service régional de l'Inventaire: HUGONNET-BERGER, Claudine, RÉVEILLON, Élisabeth, FROMAGET, Brigitte. Patrimoine hospitalier en Bourgogne. Paris : Somogy/région Bourgogne, 2011.

4. - Voir dans la base Palissy : notice PM21003279.

5. - François Friant avait épousé le 13 février 1691, à Saint-Pierre de Vézelay, Claude Anthoine (arch. dép. Yonne, état civil en ligne, BMS Saint-Pierre, 1674-1696, vue 116).

6. - Arch. mun. Vézelay, D I 53, registre des pétitions, an IV (1796). Une autre source indique qu'il acquiert la maison en 1797 du sieur Auppépin. (arch. dép. Yonne, $90 \mathrm{~J}$ ).

7. - Arch. dép. Yonne, 3 E 16/578.

8. - Plan cadastral napoléonien de 1819, section D, parcelle 402 (arch. dép. Yonne, 3 P 5829/7); l'état des sections de 1822, dans lequel Mme Flandin est dite propriétaire des parcelles D 401 (jardin), D 402 (maison) et D 403 (jardin) (arch. dép. Yonne, 3 P 5010). Les matrices mentionnent bien la mutation de la parcelle D 402 en 1848 entre Mme Gaspard et Hubert Borot suite à l'acte de vente, malheureusement sans description intérieure, du 13 avril 1844 (arch. dép. Yonne, 3 P 5011, $f^{\circ} 361$, Flandin et $f^{\circ} 82$, Hubert Borot).

9. - Arch. dép. Yonne, 40 437, ex 40461.

10. - Voir dans la base Palissy : notice PM89001862.

11. - Nous reprenons ici une partie de notre article publié dans les actes des journées d'étude des conservateurs des antiquités et objets d'art: VOTTERO, Michaël. «De la scène au foyer, la protection des théâtres en Bourgogne ». Dans BARRUOL, Agnès, CRANGA, Yves, PALOUZIÉ, Hélène (dir.). Regards sur le patrimoine des fêtes et des spectacles. Arles : Actes Sud, 2014, p. 147-151.

12. - HUGONNET-BERGER, Claudine, MAULMIN, Pascale de, SONNET, Bernard, ROSSO, Michel. Théâtres en Bourgogne, architectures du spectacle 1800-1940. Dijon : DRAC (Itinéraires du patrimoine, 124), 1996.

13. - Aux théâtres de Dijon et de Sens, inscrits en 1975 pour leurs façades et toitures, se sont ajoutés au fil des ans ceux du château du Creusot, classé en totalité en 1984, puis dans les années 1990 ceux de Nevers, Autun, Chalon-sur-Saône et Louhans, inscrits, et du château de Digoine, classé au titre des monuments historiques. L'intérêt pour ce patrimoine perdure, avec la protection du théâtre d'Auxerre en 2012 ou de l'espace des arts de Chalon-sur-Saône en 2013.

14. - M. de Jolimont. «Le théâtre de Dijon ». Le Monde dramatique, 1837, $3^{\mathrm{e}}$ année, vol. 5, p. 241.

15. - Il convient de rappeler que Werner est l'un des ébénistes les plus importants de la Restauration et remporte une médaille lors de l'Exposition des produits de l'industrie de 1827.

16. - Plusieurs ouvrages existent sur l'histoire de l'hôpital de Dijon, citons la récente plaquette publiée par l'hôpital pour fêter ses 800 ans : RIVIÈRE, Adeline (dir.). L'hôpital général de Dijon, 8 siècles d'histoire hospitalière 1204/2014. Dijon : Print-Team, 2014.

17. - Nous renvoyons à ce sujet à la plaquette de l'ANAP, Dynamisation des actifs immobiliers des établissements sanitaires médico-sociaux, tome 3: Annexes juridiques et techniques, décembre 2013. Mise en ligne: http://www.anap.fr/publications-et-outils/publications/detail/actualites/ dynamisation-des-actifs-immobiliers-des-etablissements-sanitaires-et-medico-sociaux-tome-3annexes-juridiques-et-techniques/ [consulté le 18/07/2016].

18. - Tombés dans le domaine privé de l'établissement public par l'acte de déclassement effectué par le chef d'établissement sauf s'ils sont protégés au titre des monuments historiques.

19. - Voir dans la base Palissy : notice PM89002987.

20. - Voir dans la base Palissy : notice PM89002990. 
21. - Voir dans la base Palissy : notice PM89002967.

22. - Arch. dép. Côte-d'Or, IV NIV 93 et IV NIV 102.

\section{RÉSUMÉS}

La Bourgogne, comme de nombreuses régions, conserve un certain nombre d'objets mobiliers civils protégés au titre des monuments historiques. Mairies, théâtres, administrations, hôpitaux... sont les dépositaires d'objets historiques mais également d'ensembles commandés spécifiquement pour leurs aménagements et leurs décors. Ces collections fragiles sont régulièrement étudiées et protégées par les conservations des antiquités et des objets d'art de la région. Tout en présentant quelques collections remarquables, ce texte collectif tente d'offrir une vision plurielle de ce patrimoine et des problématiques qui en découlent.

The Burgundy region, like many others, can count a certain number of civil movable objects that enjoy protection as historic monuments. Town halls, theatres, administrative buildings, hospitals... can all be places where such historic objects are preserved. They can also contain ensembles of movable objects specifically commissioned and designed for furnishing and decoration purposes. Such collections are fragile. They are regularly studied and protected by the region's curators of antiquities and art objects. Our collective text attempts an overview of some of the region's more remarkable collections and offers a plural vision of this type of heritage and some of the problems it raises.

\section{INDEX}

Mots-clés : Bourgogne, hôpital, collections publiques, mairies, théâtres, palais de justice, hôtelde-ville, ensembles, objet de mémoire, administrations, patrimoine civil

Keywords : Burgundy, hospital, public collections, town halls, theatres, courts, ensembles, objects of memory, administrations, civil heritage

\section{AUTEURS}

\section{ANNE-BÉNÉDICTE CLERT}

Conservateur délégué des antiquités et objets d'art de l'Yonne abclert@cg89.fr

\section{BRUNO FRANÇOIS}

Conservateur délégué des antiquités et objets d'art de Côte-d'Or Bruno.FRANCOIS@ars.sante.fr

\section{ADELINE RIVIÈRE}

Conservateur délégué des antiquités et objets d'art de Côte-d'Or adeline.riviere@chu-dijon.fr 


\section{MICHAËL VOTTERO}

Conservateur des monuments historiques en Bourgogne-Franche-Comté michael.vottero@culture.gouv.fr 